\title{
A multiwavelength study of the S106 region
}

\section{Structure and dynamics of the molecular gas}

\author{
N. Schneider ${ }^{1,2,3}$, R. Simon ${ }^{1,4}$, C. Kramer ${ }^{1,2}$, J. Stutzki $^{1}$, and S. Bontemps ${ }^{3}$ \\ 1 I. Physikalisches Institut, Universität zu Köln, Zülpicher Straße 77, 50937 Köln, Germany \\ 2 IRAM, BP 53, 38406 Saint Martin d'Hères, France \\ 3 Observatoire de Bordeaux, BP 89, 33270 Floirac, France \\ ${ }^{4}$ Institute for Astrophysical Research, Boston University, 725 Commonwealth Avenue, Boston, MA 02215, USA
}

Received 7 September 2001 / Accepted 15 January 2002

\begin{abstract}
The molecular cloud associated with Sharpless 106 has been studied in a variety of (sub)millimeter $\mathrm{CO}$ rotational lines on angular resolution scales from $11^{\prime \prime}$ to $80^{\prime \prime}$. We used the KOSMA $3 \mathrm{~m}$ telescope to obtain an extended ${ }^{12} \mathrm{CO} J=3 \rightarrow 2$ map, from which we calculate a total mass of $2000 M_{\odot}$ and an average density of $1.4 \times 10^{3} \mathrm{~cm}^{-3}$ for the molecular cloud. The peak intensity region around the massive young star S106 IR was observed in ${ }^{13} \mathrm{CO} J=6 \rightarrow 5$ and $3 \rightarrow 2$ with KOSMA and in isotopomeric low- $J$ CO lines with the IRAM $30 \mathrm{~m}$ telescope. A clump decomposition made for several lines yields a common clump-mass spectral index of $\alpha=1.7$, illustrating the self-similarity of the detected structures for length-scales from 0.06 to 0.9 parsec. All ${ }^{12} \mathrm{CO}$ and ${ }^{13} \mathrm{CO}$ line profiles within approximately $2^{\prime}$ around S106 IR show blue wing emission and less prominent red wing emission, partly affected by self-absorption in colder foreground material. We attribute this high-velocity emission to the ionized wind of S106 IR driving a shock into the inhomogeneous molecular cloud. We do not find evidence for a smooth or fragmented disk around S106 IR and/or an expanding ring in the observed CO emission distribution. The excitation conditions along a cut through the molecular cloud/H II region are studied with an LTE analysis (and an Escape Probability model at the position of S106 IR), using the observed CO line intensities and ratios. The kinetic gas temperature is typically $40 \mathrm{~K}$, the average density of the cloud in the core region is $9 \times 10^{3} \mathrm{~cm}^{-3}$, and the local density within the clumps is $9 \times 10^{4} \mathrm{~cm}^{-3}$. The ${ }^{13} \mathrm{CO} / \mathrm{C}^{18} \mathrm{O}$ line and column density ratios away from S106 IR reflect the natural isotopic abundance but towards the optical lobes and the cavity walls, we see enhanced ${ }^{13} \mathrm{CO}$ emission and abundance with respect to $\mathrm{C}^{18} \mathrm{O}$. This shows that selective photo-dissociation is only important close to S106 IR and in a thin layer of the cavity walls. In combination with the results from the excitation analysis we conclude that the molecular line emission arises from two different gas phases: (i) rather homogeneous, low- to medium-density, spatially extended clumps and (ii) embedded, small $(\ll 0.2 \mathrm{pc})$, high-density clumps with a low volume filling factor.
\end{abstract}

Key words. ISM: clouds - ISM: individual objects: S106 - ISM: molecules - ISM: kinematics and dynamics ISM: jets and outflows - radio lines: ISM

\section{Introduction}

The HII region S106 in Cygnus is a prominent bipolar emission nebula associated with an extended molecular cloud. The complex is most probably located at a distance of $600 \mathrm{pc}$ (Staude et al. 1983), although larger distances of 2 to $2.5 \mathrm{kpc}$ have been suggested by Reifenstein et al. (1970) and Neckel (1990, private communication). The two lobes of ionized gas seen in optical and radio continuum emission have an angular extent of $3^{\prime}$ in nearly

Send offprint requests to: N. Schneider, e-mail: schneider@observ.u-bordeaux.fr north-south direction and are separated by a dark bar of $6^{\prime} \times 2$ '.5 projected size, devoid of optical and radio continuum emission and perpendicular to the axis of symmetry of the nebula. The bar was initially interpreted as a large-scale molecular gas disk (Little et al. 1995; Bally \& Scoville 1982). Stutzki et al. (1982), however, found that their $\mathrm{NH}_{3}$ observations are inconsistent with a ring structure. Barsony et al. (1989) conclude from their molecular line data that the emission arises from a clumpy molecular cloud and found no indication for a disk-like structure.

The nebula is excited by a single O7-O9 star (Eiroa et al. 1979; Gehrz et al. 1982) referred to as S106 IR. 
The star is deeply embedded in the dark lane and drives an ionized wind with a velocity of $\approx 200 \mathrm{~km} \mathrm{~s}^{-1}$ (Br $\alpha$ observations of Simon \& Fischer 1982; model of Hippelein $\&$ Münch 1981). The magnitude of the visual extinction toward S106 IR was estimated between $12^{\mathrm{m}}$ and $21^{\mathrm{m}}$, depending on the assumed distance (Eiroa et al. 1979; Hodapp \& Rayner 1991; van den Ancker et al. 1999).

The (sub)mm dust emission in S106 was observed by Mezger et al. (1987) and Richer et al. (1993). Their maps show two emission peaks approximately $15^{\prime \prime}$ east and west of S106 IR, the latter referred to as S106 FIR. Since two clusters of $\mathrm{H}_{2} \mathrm{O}$ masers were detected at the position of S106 FIR (Stutzki et al. 1982; Furuya et al. 1999), indicating shocked material excited by a micro jet (10" lengthscale), this source was interpreted by Richer et al. (1993) as a Class 0 young stellar object. The detection of molecular material entrained in the jet, however, remains elusive.

The molecular cloud associated with the $\mathrm{H}$ in region has been studied at an angular resolution of typically $1^{\prime}$ in the lower- $J$ CO lines, $\mathrm{NH}_{3}$, and $\mathrm{CN}$ (Lucas et al. 1978; Bally \& Scoville 1982; Stutzki et al. 1982; Churchwell \& Bieging 1982). The molecular line maps in optically thick lines reveal an extended (approximately $20^{\prime} \times 25^{\prime}$ or $3.5 \mathrm{pc} \times$ $4.4 \mathrm{pc}$ at $600 \mathrm{pc}$ distance) cloud with peak emission at the position of S106 IR, whereas maps in optically thin molecular lines and mm- and submm continuum show two maxima of emission separated by $3^{\prime}$ east and west of S106 IR.

The small scale molecular cloud structure in the environment of S106 IR was studied with higher angular resolution (typically $\sim 15^{\prime \prime}$ ) single-dish maps in $\mathrm{CO}$ and CS lines (Barsony et al. 1989; Richer et al. 1993; Little et al. 1995), as well as by means of interferometric observations in HCN (Bieging 1984), CS $2 \rightarrow 1$ (Barsony et al. 1989), and $\mathrm{HCO}^{+} 1 \rightarrow 0$ (Loushin et al. 1990). On this scale, a second "bar" or "lane" of size $\approx 35^{\prime \prime} \times 7^{\text {" was de- }}$ tected and sometimes interpreted as an edge-on disk of (cold) molecular gas, responsible for collimating the ionized wind of S106 IR (Bally \& Scoville 1982; Bieging 1984; Mezger et al. 1987). Other authors, however, argue against a smooth disk of molecular material and explain the bipolarity of the nebula by either an asymmetric stellar wind (Felli et al. 1984; Barsony et al. 1989) or the presence of a very small $(30 \mathrm{AU})$ and very dense $\left(n_{\mathrm{H}}>10^{13} \mathrm{~cm}^{-3}\right)$ disk which collimates the $\mathrm{H}$ II lobes and casts an equatorial shadow in the optical and infrared seen as a narrow dark lane (Persson et al. 1988; Bally et al. 1998).

The intention of the present study is to understand the complex spatial and velocity structure observed in the molecular cloud. The S106 region with its signposts of (high mass) star formation - a bipolar H II region, a possible small or large scale disk or remnants thereof, and high velocity molecular line emission suggestive of outflow emission - makes it an ideal place to study the effects of a new born high-mass star on its parental molecular cloud. The present paper (Part I of a series) contains an analysis of low- and mid- $J$ CO lines, including their emission distribution on different size-scales, the excitation conditions, and a comparison to IR data. In Part II, we investigate the influence of the FUV radiation from S106 IR on the surrounding cloud and present a detailed study of the Photon Dominated Region (PDR).

Following a description of the data acquisition and observational parameters (Sect. 2), we present our CO observations (Sect. 3). In Sect. 4, we give the results of a clump decomposition, discuss the excitation conditions along a cut through the molecular cloud/H II region and perform an LTE and Escape Probablity analysis of the CO data. Section 5 gives a summary of the paper.

\section{Observations}

All molecular line maps obtained within the framework of this study are centered on the position of S106 IR at $\mathrm{RA}(\mathrm{B} 1950.0)=20^{\mathrm{h}} 25^{\mathrm{m}} 33.8$ and $\operatorname{DEC}(\mathrm{B} 1950.0)=$ $37^{\circ} 12^{\prime} 50^{\prime \prime}$, which will be referred to as such or the $(0,0)$ position and marked by a star in the figures throughout the paper. The technical and observing parameters of the molecular line data are summarized in Table 1.

\subsection{KOSMA ${ }^{12} \mathrm{CO}$ and ${ }^{13} \mathrm{CO} 3 \rightarrow 2,{ }^{13} \mathrm{CO} 6 \rightarrow 5$}

The ${ }^{13} \mathrm{CO} 6 \rightarrow 5$ and ${ }^{13} \mathrm{CO} 3 \rightarrow 2$ lines were observed simultaneously in 1998 February at the Kölner Observatorium für Submm-Astronomie (KOSMA) which operates a $3 \mathrm{~m}$ submm radiotelescope on Gornergrat, Switzerland (Kramer et al. 1998b). The observations were performed with a dual-channel SIS-receiver, built at the Cologne Institute, operating at $325-365 \mathrm{GHz}$ and $630-690 \mathrm{GHz}$ (Graf et al. 1998). Two acousto optical spectrometers of the Cologne group were used as backends (Schieder et al. 1989). The data were taken during a period of mean zenith opacity of 1.27 at $661 \mathrm{GHz}$ and 0.25 at $330 \mathrm{GHz}$. The observations at $330 \mathrm{GHz}$ were corrected for sideband imbalance due to an atmospheric water line at $325 \mathrm{GHz}$ as derived from an atmospheric model by Grossman (1989). The spectra were calibrated to a main beam brightness temperature scale $\left(T_{\mathrm{mb}}\right)$. Pointing was monitored simultaneously for both spectral channels using continuum cross scans of Jupiter and found to be accurate to within $15^{\prime \prime}$. The offset between the two beams was derived in the same manner and found to be $20^{\prime \prime}$ in elevation. We observed in position-switching mode with an emission free reference position at $\mathrm{RA}(\mathrm{B} 1950.0)=20^{\mathrm{h}} 24^{\mathrm{m}} 30^{\mathrm{s}}$ and $\operatorname{DEC}(\mathrm{B} 1950.0)=37^{\circ} 12^{\prime} 50^{\prime \prime}$. The average noise temperature per spectral channel of the ${ }^{13} \mathrm{CO} 6 \rightarrow 5$ data is $2.5 \mathrm{~K}$ with $4 \mathrm{~K}$ for the more noisy spectra at the northern and eastern border of the map and $1.4 \mathrm{~K}$ for the data in the central part.

A large scale map in the ${ }^{12} \mathrm{CO} J=3 \rightarrow 2$ line was taken in 1999 January using the recently implemented On-theFly (OTF) observing mode at KOSMA (Kramer et al. 1999). An area of $24^{\prime} \times 25^{\prime}$ was mapped on a fully sampled $40^{\prime \prime}$ grid. We performed scans at constant declination with a length of $10^{\prime}$, two dumps per beam-size and a spectra dump time of $4 \mathrm{~s}$. The whole area was covered once in horizontal scans during 3 observing shifts of 4 hours each. 
Table 1. Observing parameters of the molecular line data: column one and two indicate the line and transition frequency, followed by the number of points, the observing grid and Half Power Beam Width (HPBW) in arcsec; $\eta_{\mathrm{mb}}$ is the main beam efficiency, $T_{\mathrm{sys}}$ the system temperature, $\Delta v_{\text {res }}$ denotes the velocity resolution, and $\Delta T_{\mathrm{rms}}$ the average rms noise temperature per channel on a $T_{\mathrm{mb}}$ scale.

\begin{tabular}{|c|c|c|c|c|c|c|c|c|}
\hline & $\begin{array}{c}\nu \\
{[\mathrm{GHz}]}\end{array}$ & Points & Grid & HPBW & $\eta_{\mathrm{mb}}$ & $\begin{array}{c}T_{\text {sys }} \\
{[\mathrm{K}]}\end{array}$ & $\begin{array}{c}\Delta v_{\text {res }} \\
{\left[\mathrm{km} \mathrm{s}^{-1}\right]}\end{array}$ & $\begin{array}{c}\Delta T_{\mathrm{rms}} \\
{[\mathrm{K}]}\end{array}$ \\
\hline KOSMA & & & & & & & & \\
${ }^{12} \mathrm{CO} \mathrm{3 \rightarrow 2}$ & 345.796 & 1213 & $40^{\prime \prime}$ & $80^{\prime \prime}$ & 0.63 & 290 & 0.12 & 0.60 \\
${ }^{13} \mathrm{CO} \mathrm{3 \rightarrow 2}$ & 330.588 & 37 & $20^{\prime \prime}$ & $80^{\prime \prime}$ & 0.63 & 330 & 0.63 & 0.13 \\
${ }^{13} \mathrm{CO} 6 \rightarrow 5$ & 661.067 & 37 & $20^{\prime \prime}$ & $38^{\prime \prime}$ & 0.48 & 1140 & 0.23 & 2.50 \\
\hline $\mathrm{IRAM}$ & & & & & & & & \\
${ }^{12} \mathrm{CO} \mathrm{2 \rightarrow 1}$ & 230.794 & 4560 & $5.5^{\prime \prime}$ & $11^{\prime \prime}$ & 0.45 & 980 & 0.10 & 0.84 \\
${ }^{13} \mathrm{CO} \mathrm{2 \rightarrow 1}$ & 220.399 & 4560 & $5.5^{\prime \prime}$ & $11^{\prime \prime}$ & 0.45 & 650 & 0.11 & 0.47 \\
${ }^{12} \mathrm{CO} 1 \rightarrow 0$ & 115.271 & 1035 & $11^{\prime \prime}$ & $22^{\prime \prime}$ & 0.70 & 417 & 0.10 & 0.23 \\
${ }^{13} \mathrm{CO} 1 \rightarrow 0$ & 110.201 & 1035 & $11^{\prime \prime}$ & $22^{\prime \prime}$ & 0.70 & 245 & 0.11 & 0.10 \\
$\mathrm{C}^{18} \mathrm{O} 1 \rightarrow 0$ & 109.782 & 1035 & $11^{\prime \prime}$ & $22^{\prime \prime}$ & 0.70 & 269 & 0.11 & 0.11 \\
\hline
\end{tabular}

The area of peak CO emission was included in each of the shifts, showing that the deviations in intensity between the maps are less than $10 \%$. The data were taken with the same front- and backends used in 1998. The pointing accuracy was of the order of $10^{\prime \prime}$, derived from continuum cross scans of Jupiter.

\subsection{Isotopomeric IRAM CO $2 \rightarrow 1$ and $1 \rightarrow 0$ lines}

In 1997 June, we observed the ${ }^{12} \mathrm{CO}$ and ${ }^{13} \mathrm{CO} J=2 \rightarrow 1$, and the ${ }^{12} \mathrm{CO},{ }^{13} \mathrm{CO}$, and $\mathrm{C}^{18} \mathrm{O} J=1 \rightarrow 0$ lines with the IRAM $30 \mathrm{~m}$ telescope in its OTF mapping mode. The spectral lines were recorded simultaneously under uniform weather conditions (atmospheric opacity of 0.4 at $230 \mathrm{GHz}$ ) prevailing over several days. We used the IRAM facility receivers and autocorrelator which was adapted in spectral resolution and bandwidth so that all CO lines have a similar velocity resolution of $\sim 0.1 \mathrm{~km} \mathrm{~s}^{-1}$. Pointing and focus were checked every 2 hours. The pointing accuracy was found to be better than $4^{\prime \prime}$ and the receivers were aligned to within $2^{\prime \prime}$.

The OTF mapping was started with a horizontal coverage (scans in RA) of an $8^{\prime} \times 4^{\prime}$ large area with 6 dumps per beam-size and a dump time of $2 \mathrm{~s}$. We then proceeded to cover the same area with vertical scans to obtain complete, but independent, maps of the whole region and to reduce smearing effects and fluctuations in the intensity calibration. The final maps in each molecular line are the weighted average of all data obtained. The $1 \rightarrow 0$ maps show no obvious scanning effects or calibration problems, in contrast to the higher angular resolution maps of the $2 \rightarrow 1$ line, where we see slight scanning effects in the form of low intensity horizontal and vertical stripes.

Since the observed molecular cloud region is extended, the contribution from the $170^{\prime \prime}$ error beam of the IRAM $30 \mathrm{~m}$ telescope at high frequencies, with a fraction of 0.31 of the total intensity on the Moon (Garcia-Burillo et al. 1993; Greve et al. 1998), cannot be neglected. The IRAM $2 \rightarrow 1$ spectra were therefore reduced in intensity by $25 \%$, a value which is somewhat arbitrary since the absolute error beam contribution varies for each position observed. A more sophisticated procedure would be to correct for the error beam contribution by using molecular line maps obtained at smaller telescopes, as described in Schneider et al. (1998).

\section{Results}

\subsection{Low angular resolution $\mathrm{CO}$ maps: ${ }^{12} \mathrm{CO}$ and ${ }^{13} \mathrm{CO} 3 \rightarrow 2$, and ${ }^{13} \mathrm{CO} 6 \rightarrow 5$}

\subsubsection{Large scale ${ }^{12} \mathrm{CO} 3 \rightarrow 2$ emission}

Figure 1 shows the line integrated $\left(-8\right.$ to $\left.6 \mathrm{~km} \mathrm{~s}^{-1}\right)$ ${ }^{12} \mathrm{CO} 3 \rightarrow 2$ map at $80^{\prime \prime}$ resolution, overlaid on a $2 \mu \mathrm{m}$ image from $2 \mathrm{MASS}^{1}$. The $\mathrm{CO}$ emission distribution is similar to that of the slightly smaller ${ }^{12} \mathrm{CO} 1 \rightarrow 0$ map $\left(50^{\prime \prime}\right.$ angular resolution) by Bally \& Scoville (1982). The molecular gas with a typical main beam brightness temperature in the CO $3 \rightarrow 2$ line above $10 \mathrm{~K}$ extends on a size-scale of at least $24^{\prime} \times 25^{\prime}(4.2 \times 4.4 \mathrm{pc})$. From Fig. 1, the cloud can be divided into a well defined high intensity core around the young star S106 IR $\left(T_{\mathrm{mb}}=20\right.$ to $\left.30 \mathrm{~K}\right)$ and a more diffuse, lower intensity $\left(T_{\mathrm{mb}} \leq 15 \mathrm{~K}\right)$ region to the southwest.

The channel maps of Fig. 2 reveal the velocity structure of the cloud and isolate individual cloud fragments: At low velocities $\left(-6\right.$ to $\left.-2 \mathrm{~km} \mathrm{~s}^{-1}\right)$, the typical doublepeak structure, which was also seen in the ${ }^{13} \mathrm{CO} 1 \rightarrow 0$ map (50" resolution like the ${ }^{12} \mathrm{CO} 1 \rightarrow 0$ map) of Bally \& Scoville (1982), is apparent. With increasing velocity, the emission merges into one peak slightly shifted to the southwest of S106 IR and a second, pronounced peak (associated with S106 South) establishes roughly $4^{\prime}$ further south to the main peak but disappears at higher velocities (emission between -2.3 and $1 \mathrm{kms}^{-1}$ ). Between -3 and $4 \mathrm{~km} \mathrm{~s}^{-1}$, the lower intensity emission of the extended

\footnotetext{
1 The Two Micron All Sky Survey (2MASS) is a joint project of the University of Massachusetts and the Infrared Processing and Analysis Center/California Institute of Technology, funded by the National Aeronautics and Space Administration and the National Science Foundation.
} 


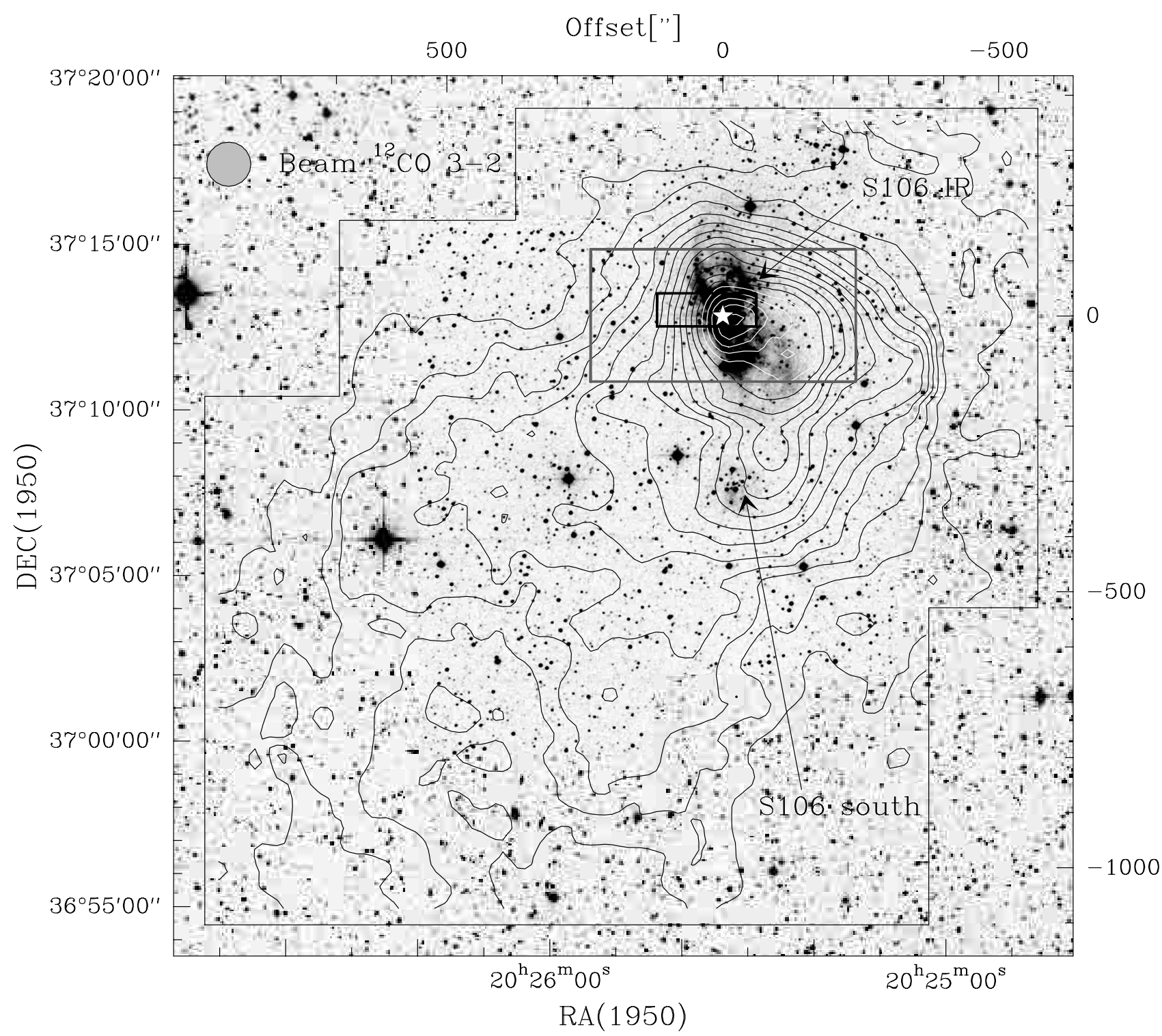

Fig. 1. Contour lines of the KOSMA ${ }^{12} \mathrm{CO} J=3 \rightarrow 2$ map ( $80^{\prime \prime}$ angular resolution) overlaid on a $2 \mu \mathrm{m}$ image of S106 taken from 2MASS show the large scale morphology and extent of the molecular cloud. The line was integrated over the velocity interval -8 to $6 \mathrm{~km} \mathrm{~s}^{-1}$. Contour levels range from $5.5(3 \sigma)$ to $181.5 \mathrm{~K} \mathrm{~km} \mathrm{~s}^{-1}$ in steps of $11 \mathrm{~K} \mathrm{~km} \mathrm{~s}^{-1}(6 \sigma)$ (temperatures are on a main beam brightness scale) and show a maximum at the position of the exciting star and strong infrared source S106 IR which is marked by a star symbol. The nebula itself shows a bipolar structure with a northern and southern lobe on a size-scale of a few arcminutes. The regions observed in tracers other than ${ }^{12} \mathrm{CO} J=3 \rightarrow 2$ are marked by rectangles (IRAM CO-lines: solid grey line, KOSMA ${ }^{13} \mathrm{CO} 6 \rightarrow 5$ : solid black line). South of S106 IR, a newly detected embedded cluster (S106 South) is indicated by an arrow.

molecular cloud is detected southeast of S106 IR, forming "streamers" of molecular gas and giving the cloud a cometary shaped structure.

\subsubsection{The star formation activity in S106}

The primary star formation site in S106 is located within a radius of 1.7 around S106 IR and contains $\sim 160 \mathrm{IR}$ sources (Hodapp \& Rayner 1991). It is coincident with the prominent peak in CO emission already discussed in Sect. 3.1.1. The second, weaker CO peak south of
S106 IR harbours a small IR cluster and nebulosity (S106 south) evident in the 2MASS image at $2 \mu \mathrm{m}$ : weak extended emission is apparent $5^{\prime}$ south of S106 IR (see Fig. 1). Since this newly recognized nebula also corresponds to a slight enhancement in the stellar density distribution of the brightest 2MASS $2 \mu \mathrm{m}$ sources (sources with $\left.K_{\mathrm{S}}<11.0-0.52 \times\left(J-K_{\mathrm{s}}\right)\right)$; e.g., Bontemps $(2001)$, Knödlseder (2000), we interpret the nebula as a signpost for a secondary site of star formation in S106. A couple of (most likely) young stars seen by 2MASS are the brightest members of a probable embedded cluster 


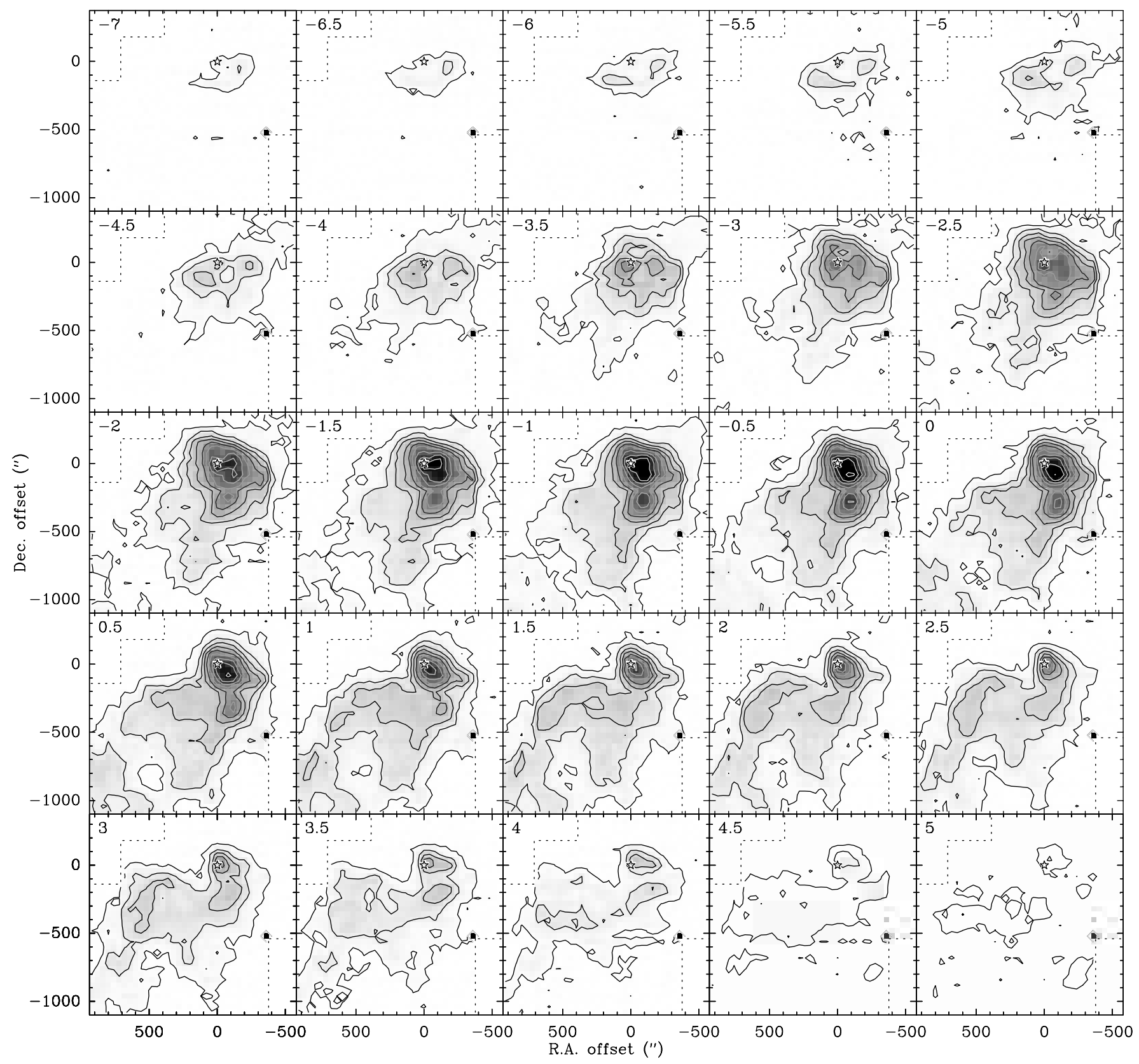

Fig. 2. Channel maps of the "On-the-Fly" observations of the ${ }^{12} \mathrm{CO} 3 \rightarrow 2$ line emission, obtained at KOSMA. Center velocities of the channels are indicated in the upper left corner of each panel and increase in steps of $0.5 \mathrm{~km} \mathrm{~s}^{-1}$. Contour lines are drawn from 1 to $28 \mathrm{~K} \mathrm{~km} \mathrm{~s}^{-1}$ in steps of $1 \mathrm{~K} \mathrm{~km} \mathrm{~s}^{-1}$. The $3 \sigma$ level is $0.95 \mathrm{~K} \mathrm{~km} \mathrm{~s}^{-1}$. The star marks the position of S106 IR. The dotted lines mark the boundaries of the region observed.

referred to here as S106 South. Finally we note that two mid-IR sources observed by MSX (Price et al. 1998) are also found in S106 South: MSX5C_G076.3045-00.6721 and MSX5C_G076.3116-00.6582. They are detected at a 1 to 2 Jy level between 8 and $15 \mu \mathrm{m}$ and both have a counterpart in the 2MASS point source catalog. Their IR colors (calculated between 2 and $15 \mu \mathrm{m}$ ) are typical of Class I young stellar objects as defined by Lada (1987), and their mid-IR fluxes at $600 \mathrm{pc}$ are similar to the flux of the brightest protostar of the $\rho$ Ophiuchi cloud (EL29; Bontemps et al. 2001) suggesting they are as luminous as EL29, i.e. of the order of 20 to $30 \mathrm{~L} \odot$.

\subsection{3. ${ }^{13} \mathrm{CO} J=6 \rightarrow 5$ emission}

The central area $\left(3^{\prime} \times 1^{\prime}\right)$ around S106 IR (see Fig. 1) was mapped in ${ }^{13} \mathrm{CO} 6 \rightarrow 5$ and is shown in Fig. 3, overlaid on a map of [C II] $158 \mu \mathrm{m}$ emission obtained at the $\mathrm{KAO}$ (Schneider et al. 1999a, 1999b; Paper II). The ${ }^{13} \mathrm{CO} 6 \rightarrow 5$ emission extends outside the mapped region in all directions except to the east. It closely follows the [C II] emission distribution showing that this mid- $J$ CO line traces the warm molecular component of PDRs. 


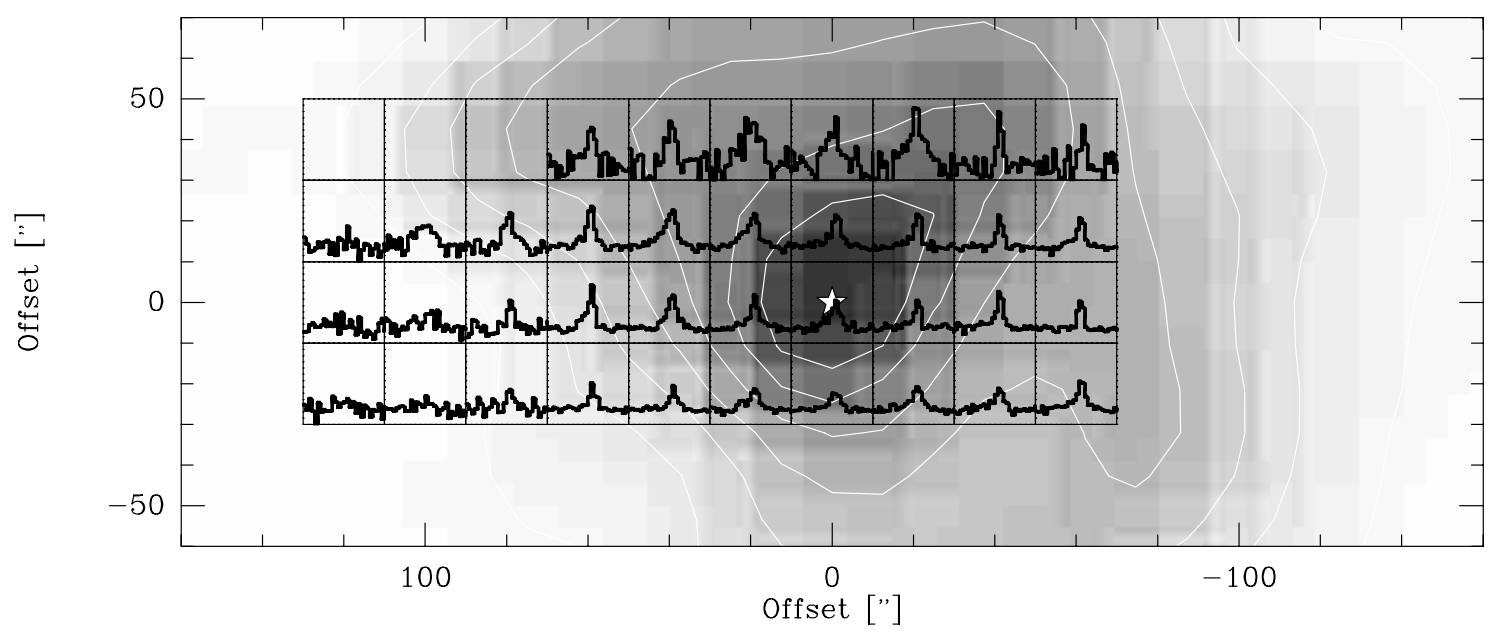

Fig. 3. The ${ }^{13} \mathrm{CO} J=6 \rightarrow 5$ spectra ( $38^{\prime \prime}$ angular resolution), obtained with KOSMA, are overlaid on a grey scale plot of the [C II] $158 \mu \mathrm{m}$ emission at 55" resolution (Schneider et al. 1999a, 1999b, Paper II). The velocity range of the spectra is -15 to $+10 \mathrm{~km} \mathrm{~s}^{-1}$, the main beam brightness temperature scale is -4 to $+19 \mathrm{~K}$. The star marks the position of S106 IR.

The peak intensity of around $13 \mathrm{~K}$, which is equivalent to a Rayleigh-Jeans corrected temperature of $26 \mathrm{~K}$, is found at offset $\left(60^{\prime \prime}, 0\right)$, a region with significant [C $\left.\mathrm{II}\right]$ emission, whereas in the outskirts of the PDR (at $80^{\prime \prime}, 0$ ), indicated by a strong gradient of decreasing [ $[\mathrm{CII}]$ intensity, the $\mathrm{CO}$ line intensity is reduced to about $6 \mathrm{~K}$. The $\mathrm{CO}$ intensity also drops (to $8 \mathrm{~K}$ ) at the central position $(0,0)$ but increases again to $11 \mathrm{~K}$ at $\left(-40^{\prime \prime}, 0\right)$.

\subsubsection{A comparison of $\mathrm{CO}$ spectra at the position of S106 IR}

Figure 4 shows ${ }^{12} \mathrm{CO}$ and ${ }^{13} \mathrm{CO} 3 \rightarrow 2$, and ${ }^{13} \mathrm{CO} 6 \rightarrow 5$ spectra at the position of S106 IR. All CO lines are strongest at the velocity of the ambient cloud $\left(-1.5 \mathrm{~km} \mathrm{~s}^{-1}\right)$. The line profiles are non-Gaussian, indicating a composite of several velocity components overlapping along the lineof-sight and/or highly turbulent gas. As we will see in Sect. 3.2, the line profiles turn out to be even more complex in the higher angular resolution IRAM observations. Although some of this fine structure is blended in the KOSMA data, we can still identify two major components at $-1.5 \mathrm{~km} \mathrm{~s}^{-1}$ and $2 \mathrm{~km} \mathrm{~s}^{-1}$. The typical line widths of these components are rather narrow $\left(2 \mathrm{~km} \mathrm{~s}^{-1}\right)$ making shock heating of the corresponding gas unlikely. A two component Gaussian fit to the ${ }^{12} \mathrm{CO} 3 \rightarrow 2$ line reveals strong excess blue wing emission extending approximately between -5 and $-10 \mathrm{~km} \mathrm{~s}^{-1}$. This finding confirms the detection of blue wing emission by other authors, e.g., in the [C I] $492 \mathrm{GHz}$ line and the $\mathrm{C}^{18} \mathrm{O} J=3 \rightarrow 2$ and $2 \rightarrow 1$ lines by Little et al. (1995). The fit reveals that there is also some, less prominent, excess redshifted emission at positive velocities between 2 and $6 \mathrm{~km} \mathrm{~s}^{-1}$. We can identify this red wing emission also in the ${ }^{13} \mathrm{CO} 3 \rightarrow 2$ spectra which is not as obvious in ${ }^{13} \mathrm{CO} 6 \rightarrow 5$ (there, some redshifted emission appears between approximately 1 and $3 \mathrm{~km} \mathrm{~s}^{-1}$ ).
In contrast, the blue wing emission (down to $-10 \mathrm{~km} \mathrm{~s}^{-1}$ ) is strong in all tracers. The fact that we see this high-velocity emission even in the ${ }^{13} \mathrm{CO} 6 \rightarrow 5$ line indicates that it arises in high column density and warm gas.

\subsection{High angular resolution $\mathrm{CO}$ maps: Isotopomeric $\mathrm{CO} \mathrm{J}=2 \rightarrow 1$ and $\mathrm{J}=1 \rightarrow 0$}

\subsubsection{The spatial and velocity distribution of molecular gas in the cloud core region}

The molecular cloud core around S106 IR and the extended PDR region, an area of $8^{\prime} \times 4^{\prime}$ (indicated in Fig. 1), was observed in the ${ }^{12} \mathrm{CO},{ }^{13} \mathrm{CO}$, and $\mathrm{C}^{18} \mathrm{O} 1 \rightarrow 0$, and ${ }^{12} \mathrm{CO}$ and ${ }^{13} \mathrm{CO} 2 \rightarrow 1$ lines at the IRAM $30 \mathrm{~m}$ telescope. Figure 5 shows grey scale plots of the line integrated emission of these lines.

The molecular gas is concentrated within two major emission regions $50^{\prime \prime}$ east and $100^{\prime \prime}$ west of S106 IR already visible in the ${ }^{12} \mathrm{CO} 3 \rightarrow 2$ map (Fig. 2). In between, the lobes of the ionized gas in the $\mathrm{H}$ II region are clearly outlined by a lack of molecular line emission north and south of S106 IR. The transition zones between molecular cloud and $\mathrm{H}$ II region are marked by a strong $\mathrm{CO}$ emission gradient. We see sharp borders, i.e. edge-on PDRs, running northeast to southwest in the ${ }^{13} \mathrm{CO}$ and $\mathrm{C}^{18} \mathrm{O} 1 \rightarrow 0$ maps on both sides of the cavity. The stronger eastern CO peak, referred to as "East Clump" (Little et al. 1995), connects to the western part of the cloud via a bridge of emission crossing the (sub)mm continuum source S106 FIR (15" west of S106 IR). S106 FIR is not resolved as an individual peak due to beam dilution and is best visible in the ${ }^{13} \mathrm{CO} 2 \rightarrow 1$ map.

There are significant morphological differences between the maps which mainly reflect differences in excitation and optical depth. ${ }^{12} \mathrm{CO}$ and ${ }^{13} \mathrm{CO} \quad 1 \rightarrow 0$ and ${ }^{13} \mathrm{CO} 2 \rightarrow 1$ have an emission maximum at the edge of the 


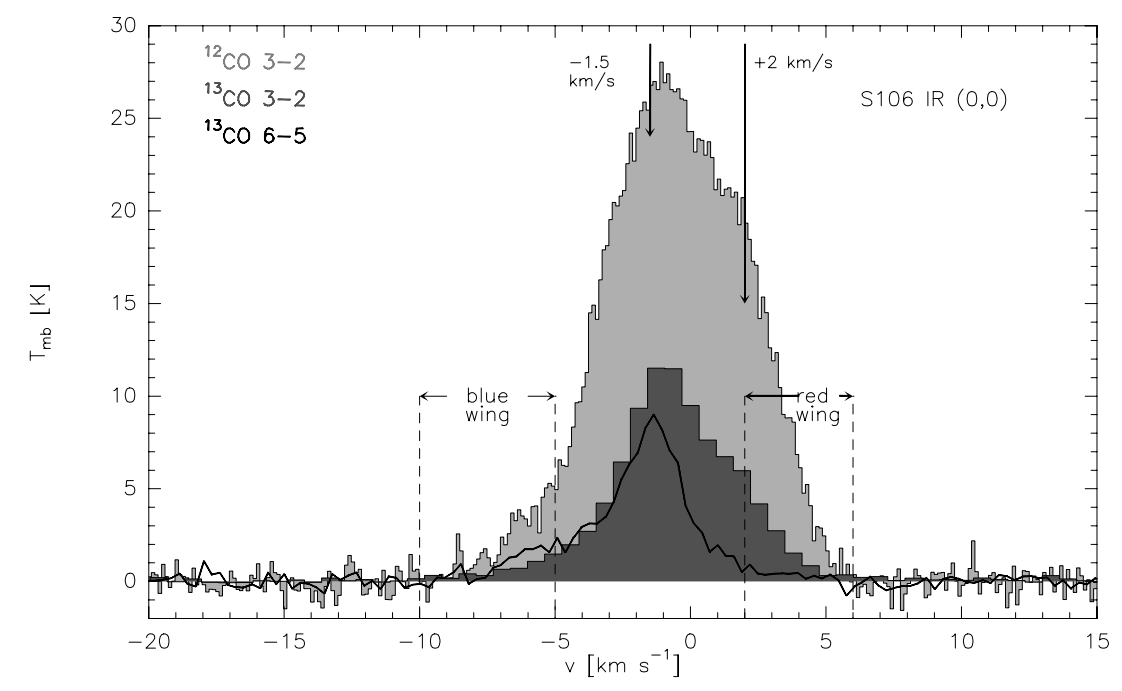

Fig. 4. Comparison of isotopomeric CO spectra at the same angular resolution of $80^{\prime \prime}$ (the $6 \rightarrow 5$ data were smoothed to the resolution of the $3 \rightarrow 2$ observations) at the position of S106 IR. The long-dashed lines indicate the velocity intervals of red and blue wing emission.

East Clump and trace the temperature profile in the cloud core with a maximum close to the embedded star S106 IR. The transitions of the rarer isotopomers ${ }^{13} \mathrm{CO}$ and $\mathrm{C}^{18} \mathrm{O}$ show more contrast than the ${ }^{12} \mathrm{CO}$ maps and uncover the column density distribution of the bulk of the colder material in the cloud away from S106 IR. These optically thinner lines show a more north-south oriented distribution and the East Clump in $\mathrm{C}^{18} \mathrm{O}$ even breaks up into several distinct clumps along the cavity walls.

Figure 6 shows channel maps of the ${ }^{12} \mathrm{CO}$ and ${ }^{13} \mathrm{CO} 2 \rightarrow 1$ emission which impressively reveal the complex spatial and velocity structure of the molecular cloud. We distinguish five velocity intervals, reflecting the main dynamical features of the molecular gas:

-9 to $-4 \mathrm{~km} \mathrm{~s}^{-1}$

This blueshifted high-velocity emission, already apparent in the lower angular resolution CO spectra (see Sect. 3.1), is resolved to be confined to a small region around S106 IR, coincident with the edge of the East Clump. More diffuse and extended emission is also visible approximately $120^{\prime \prime}$ west and $150^{\prime \prime}$ southeast of S106 IR.

\section{-3.5 to $-2 \mathrm{~km} \mathrm{~s}^{-1}$}

The high-velocity peak emission at S106 IR starts to merge with the bulk emission of the cloud. In this velocity range, the "dark lane" is best visible, evident as a narrow tongue of emission close to S106 IR in approximately east-west direction.

\section{-1.5 to $+0.5 \mathrm{~km} \mathrm{~s}^{-1}$}

This velocity interval represents the bulk emission of the molecular cloud. The prominent double-peak morphology of the cloud is evident, with an emission distribution elongated north-south along both sides of the cavity walls of the optical lobes. Again, the $\mathrm{H}$ II region is nicely outlined by a lack of molecular emission. The two peak emission regions east and west of S106 IR are linked via a bridge of weaker emission crossing S106 IR, best seen at -1.5 to $-0.5 \mathrm{~km} \mathrm{~s}^{-1}$. At the tips of the southern and northern optical lobe, $\mathrm{CO}$ emission traces swept-up material at the end of the north-south flow from S106 IR. This gas is not blue- or redshifted from the velocity of the ambient cloud since the inclination angle of the two optical lobes is small, $14^{\circ}$ with respect to the plane of the sky, and within the range of the aperture angle of the lobes $\left(2 \Phi \sim 35^{\circ}\right.$, Solf \& Carsenty 1982).

The observed morphology and kinematics are thus fully consistent with the picture of a cavity created by S106 IR whose radiation and ionized wind sweep-up material from the cavity walls marked by the two lobes of the $\mathrm{H}$ II region and at the extreme ends of the flow.

+1 to $+2 \mathrm{~km} \mathrm{~s}^{-1}$

The double-peak structure of the cloud breaks down and the emission merges into a more diffuse extended plateau at S106 IR where the molecular emission traces the redshifted component of the stellar wind hitting the backside of the cavity walls.

+2 to $+6 \mathrm{~km} \mathrm{~s}^{-1}$

At these redshifted velocities, we identify two emission regions (mainly in ${ }^{12} \mathrm{CO}$ ). The first is centered on $\mathrm{S} 106 \mathrm{IR}$ (at $2.5 \mathrm{~km} \mathrm{~s}^{-1}$ ), before shifting north for $v=4$ to $6 \mathrm{~km} \mathrm{~s}^{-1}$. The second appears at $3.5 \mathrm{~km} \mathrm{~s}^{-1}$, approximately $120^{\prime \prime}$ west of S106 IR, but disappears at $5.0 \mathrm{~km} \mathrm{~s}^{-1}$. The CO emission distribution in this velocity range appears not as strong and confined as in the blueshifted counterpart probably because the densest part of the East clump is located at the near, blueshifted side of S106 IR.

\subsubsection{Self-absorption effects in spectral line profiles}

The variety in the $\mathrm{CO}$ emission distribution is not only due to the complex kinematics of the molecular gas, but also influenced by self-absorption effects, as the following analysis of the spectral line profiles illustrates.

Figure 7 displays spectra selected along a cut at constant Declination through S106 IR. In all ${ }^{12} \mathrm{CO}$ spectra, 

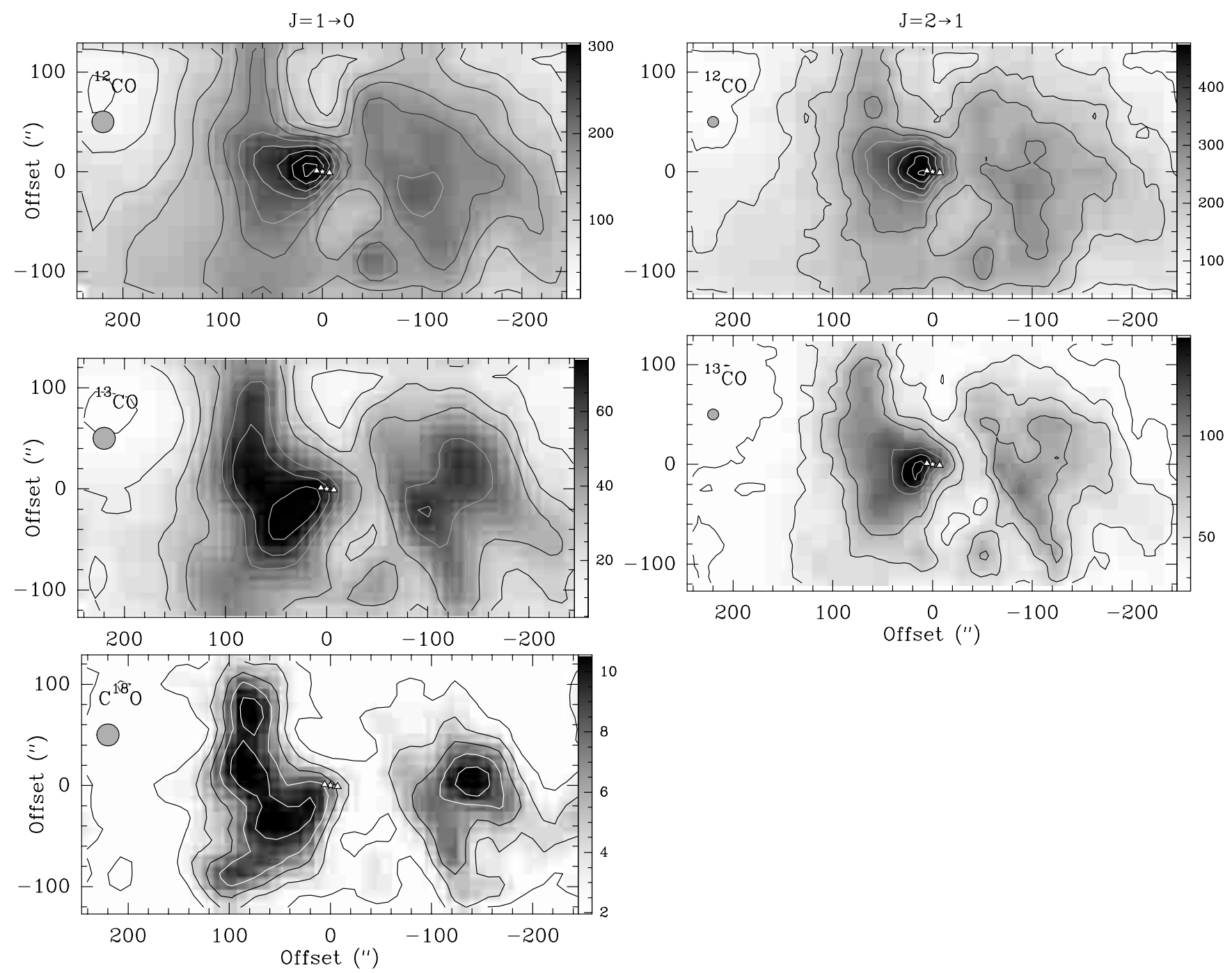

Fig. 5. Maps of the line integrated CO $1 \rightarrow 0$ (left) and $2 \rightarrow 1$ (right) emission in the velocity range -10 to $7 \mathrm{~km} \mathrm{~s}^{-1}$. The isotopomers and beam-sizes are indicated on the left hand side of each panel. The wedge on the right hand side indicates the integrated intensity in $\mathrm{K} \mathrm{km} \mathrm{s}^{-1}$. The contour ranges are as follows (in the notation start/end/step): ${ }^{12} \mathrm{CO} 1 \rightarrow 030 / 330 / 30 \mathrm{~K}$ $\mathrm{km} \mathrm{s}^{-1}\left(3 \sigma=9.8 \mathrm{~K} \mathrm{~km} \mathrm{~s}^{-1}\right),{ }^{12} \mathrm{CO} 2 \rightarrow 170 / 520 / 50 \quad \mathrm{~K} \mathrm{~km} \mathrm{~s}^{-1}\left(3 \sigma=35 \mathrm{~K} \mathrm{~km} \mathrm{~s}^{-1}\right),{ }^{13} \mathrm{CO} 1 \rightarrow 0 \quad 5 / 75 / 10 \quad \mathrm{~K} \mathrm{~km} \mathrm{~s}{ }^{-1}(3 \sigma=$ $\left.4.7 \mathrm{~K} \mathrm{~km} \mathrm{~s}^{-1}\right),{ }^{13} \mathrm{CO} 2 \rightarrow 120 / 160 / 20 \quad \mathrm{~K} \mathrm{~km} \mathrm{~s}^{-1}\left(3 \sigma=23.5 \mathrm{~K} \mathrm{~km} \mathrm{~s}^{-1}\right), \mathrm{C}^{18} \mathrm{O} 1 \rightarrow 02 / 10 / 2 \quad \mathrm{~K} \mathrm{~km} \mathrm{~s}^{-1}\left(3 \sigma=2 \mathrm{~K} \mathrm{kms}^{-1}\right)$. The positions of S106 IR and the submm continuum peaks identified by Mezger et al. (1987) and Richer et al. (1993) (the western peak is S106 FIR) are marked as a star and as triangles.

we see broad blue wing emission approximately between -10 and $-5 \mathrm{~km} \mathrm{~s}^{-1}$, which is strongest at the positions of S106 IR $(0,0)$ and the East clump $\left(20^{\prime \prime}, 0\right)$. This highvelocity wing is also seen in ${ }^{13} \mathrm{CO} 2 \rightarrow 1$ and $1 \rightarrow 0$ at position $(0,0)$ and, less prominent, at $\left(20^{\prime \prime}, 0\right)$. The ratio of the integrated line intensity of ${ }^{12} \mathrm{CO}$ and ${ }^{13} \mathrm{CO} 2 \rightarrow 1$ in this velocity range is 8 for the $(0,0)$ position and 12 towards $\left(20^{\prime \prime}, 0\right)$, indicating that the ${ }^{13} \mathrm{CO}$ emission is optically thin.

The redshifted counterpart of the blue wing emission is less pronounced but nevertheless visible in all ${ }^{12} \mathrm{CO} 2 \rightarrow 1$ spectra at velocities higher than $3 \mathrm{~km} \mathrm{~s}^{-1}$. Around $3 \mathrm{~km} \mathrm{~s}^{-1}$, the ${ }^{12} \mathrm{CO}$ spectra show a dip in intensity that gets filled in with emission in the corresponding ${ }^{13} \mathrm{CO}$ spectra, causing the ${ }^{12} \mathrm{CO} /{ }^{13} \mathrm{CO}$ ratio to drop to values around 1 or lower for this velocity range. The lack of ${ }^{12} \mathrm{CO}$ emission is hence due to self-absorption of the optically thick ${ }^{12} \mathrm{CO}$ line in cold foreground material, presumably associated with the extended molecular cloud. Figure 8 shows the blue- and redshifted emission distributions and the positions where the ${ }^{12} \mathrm{CO} /{ }^{13} \mathrm{CO}$ ratio is lower than unity. We see significant self-absorption in the western part of the region (an area of $100^{\prime \prime} \times 100^{\prime \prime}$ centered on the position $-120^{\prime \prime}$, 0) which explains, at least partly, why prominent red wing emission in S106 was not previously detected.

The ${ }^{12} \mathrm{CO}$ spectra close to S106 IR, at $(0,0),\left(20^{\prime \prime}, 0\right)$ and $\left(-20^{\prime \prime}, 0\right)$, reveal a dip in the line center at $-1 \mathrm{~km} \mathrm{~s}^{-1}$. Since the ${ }^{13} \mathrm{CO}$ and $\mathrm{C}^{18} \mathrm{O} 1 \rightarrow 0$ lines peak at the velocity of the dip, the optically thick ${ }^{12} \mathrm{CO}$ is hence clearly also 
${ }^{12} \mathrm{CO} \quad 2 \rightarrow 1$

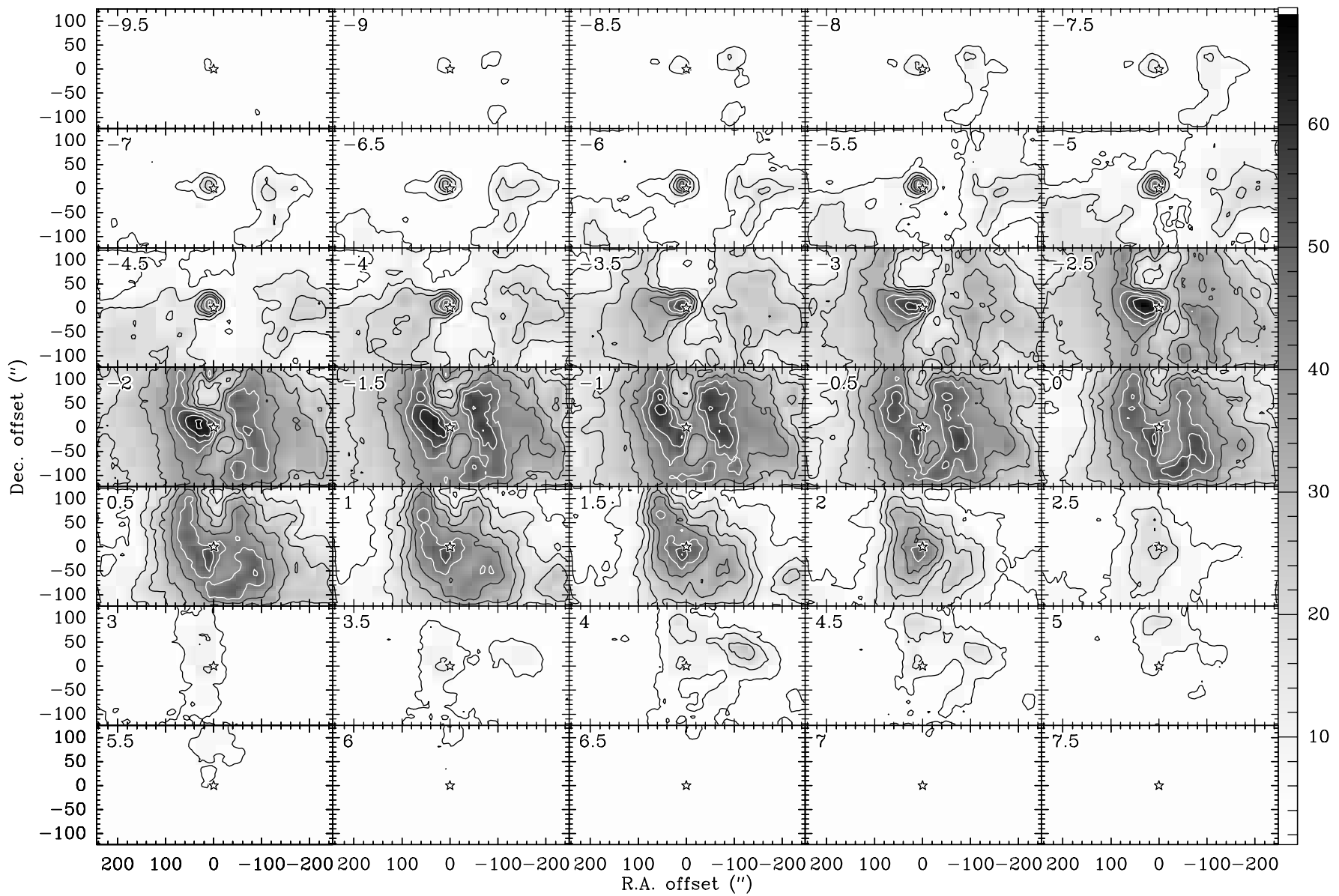

${ }^{13} \mathrm{CO} 2 \rightarrow 1$

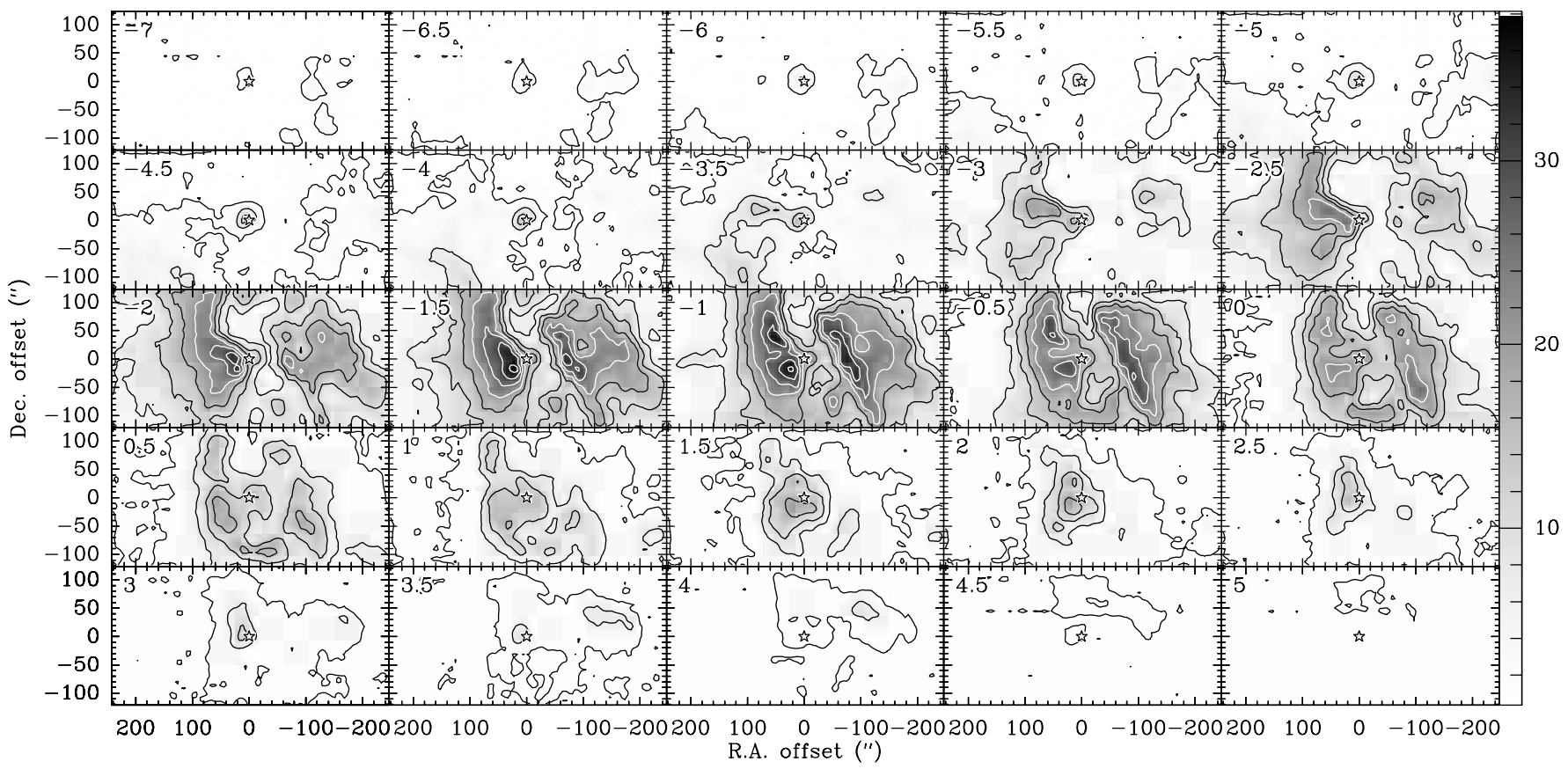

Fig. 6. Channel maps of ${ }^{12} \mathrm{CO} 2 \rightarrow 1$ (top) and ${ }^{13} \mathrm{CO} 2 \rightarrow 1$ (bottom) observations. The contour levels are 4 to $68 \mathrm{~K} \mathrm{~km} \mathrm{~s}{ }^{-1}$ by $8 \mathrm{~K} \mathrm{~km} \mathrm{~s}^{-1}\left(3 \sigma=3.7 \mathrm{~K} \mathrm{~km} \mathrm{~s}^{-1}\right)$ for ${ }^{12} \mathrm{CO}$ and 1 to $36 \mathrm{~K} \mathrm{~km} \mathrm{~s}^{-1}$ by $5 \mathrm{~K} \mathrm{~km} \mathrm{~s}^{-1}\left(3 \sigma=1.1 \mathrm{~K} \mathrm{~km} \mathrm{~s}^{-1}\right)$ for ${ }^{13} \mathrm{CO}$. The wedges on the right hand side indicate the integrated intensity in $\mathrm{K} \mathrm{km} \mathrm{s}^{-1}$. The velocity steps are $0.5 \mathrm{~km} \mathrm{~s}^{-1}$, as indicated in the upper left corner of each panel. The star marks the position of S106 IR. 


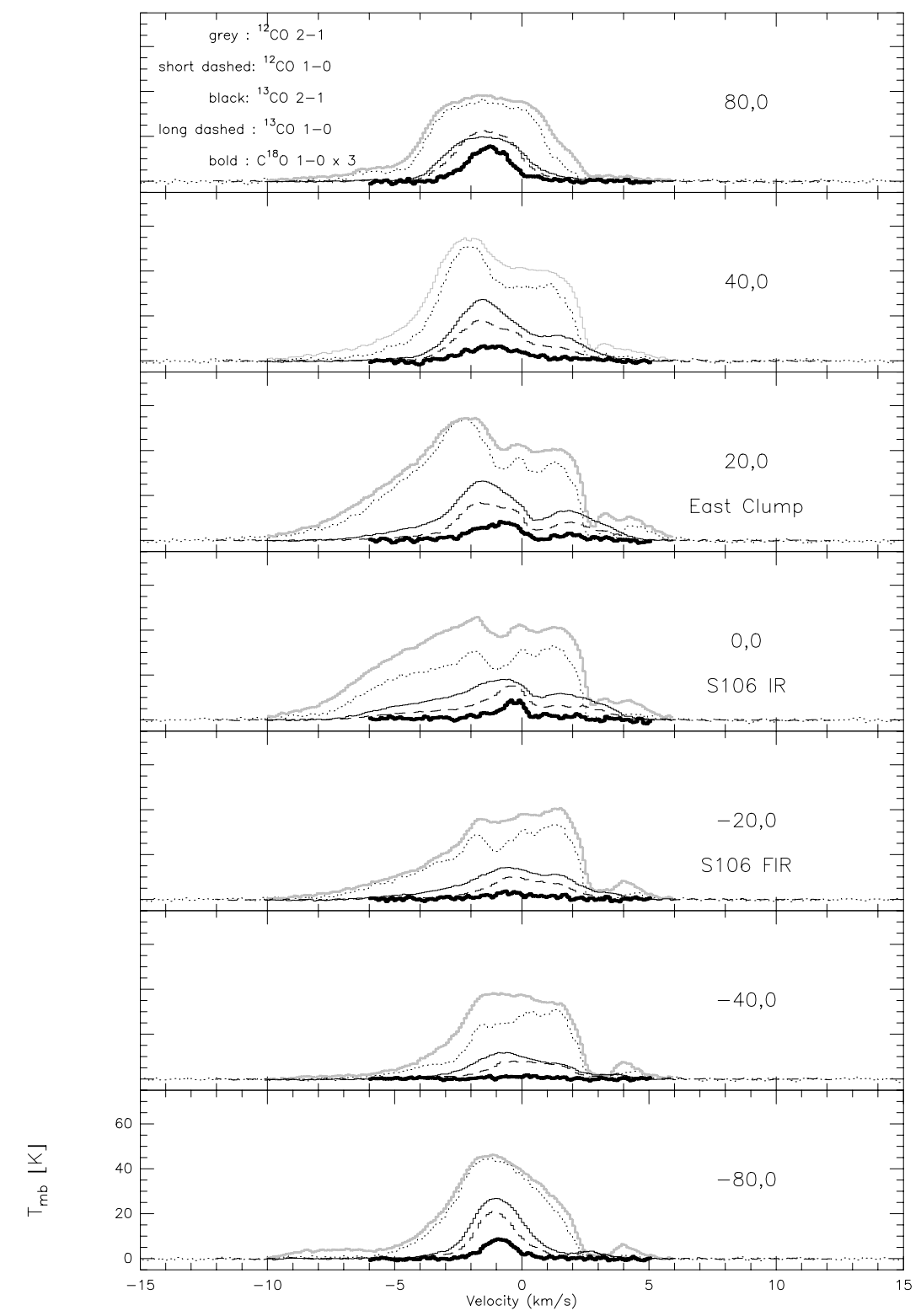

Fig. 7. Isotopomeric CO $1 \rightarrow 0$ and $2 \rightarrow 1$ spectra with an angular resolution of $20^{\prime \prime}$ are displayed along a cut through S106 IR in east-west direction. The positions are given as offsets in arcsec from S106 IR. The $\mathrm{C}^{18} \mathrm{O} 1 \rightarrow 0$ line intensity is scaled up by a factor of 3. The line profiles show the effects of outflow emission and self-absorption.

self-absorbed at velocities around $-1 \mathrm{~km} \mathrm{~s}^{-1}$. The two velocity components at $-1 \mathrm{kms}^{-1}$ and $+2 \mathrm{kms}^{-1}$ in the $\mathrm{C}^{18} \mathrm{O} 1 \rightarrow 0$ and ${ }^{13} \mathrm{CO} 2 \rightarrow 1$ spectra at $\mathrm{S} 106 \mathrm{IR}$ and the East Clump are due to a true line splitting, as opposed to self-absorption, which confirms the finding of Little et al. (1995).

\subsubsection{Other velocity features}

In order to identify other velocity signatures, e.g., rotation, we display the data cubes as position-velocity channel maps in Fig. 9. First, the most prominent features already introduced in the preceeding sections are again clearly visible: the typical double-peak structure of the molecular cloud with peak ${ }^{12} \mathrm{CO}$ and ${ }^{13} \mathrm{CO}$ emission east and west of S106 IR as well as the prominent "East Clump". Its close correlation with the highly blueshifted emission suggests that the parts of the East Clump closest to S106 IR are being dispersed by the stellar wind. We also see the blue and red high-velocity wing emission, which show the highest degree of apparent confinement around S106 IR (panels corresponding to $0^{\prime \prime}$ and $20^{\prime \prime}$ offset in declination). The region of self-absorption in the red wing of the ${ }^{12} \mathrm{CO}$ line discussed in the previous section is nicely outlined by the contours of the ${ }^{13} \mathrm{CO}$ emission in the panels with Declination offsets $-45^{\prime \prime}$ to $65^{\prime \prime}$.

There is no signature of systematic rotation of the cloud or of a smooth disk in the position-velocity plots. Instead, the velocities increase from west and east towards the center. The eastern gradient is strongest 


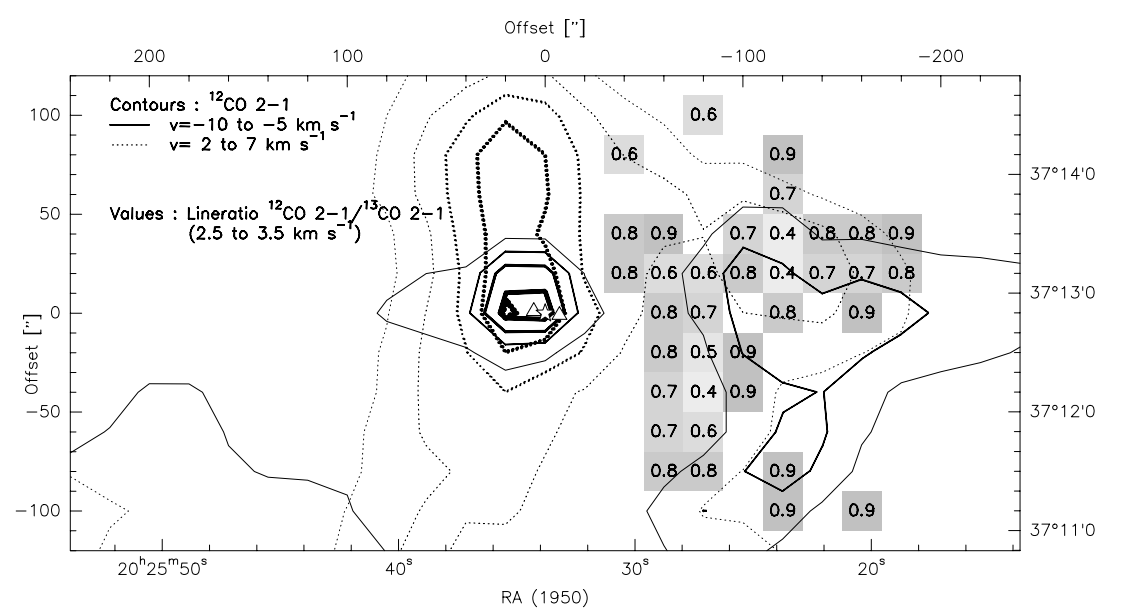

Fig. 8. Blue and red wing emission and self-absorption in the red wing component. Positions where the ${ }^{12} \mathrm{CO} 2 \rightarrow 1 /{ }^{13} \mathrm{CO} 2 \rightarrow 1$ line ratio in the velocity interval between 2.5 and $3.5 \mathrm{~km} \mathrm{~s}^{-1}$ is lower than 1 (indicating self-absorption of the ${ }^{12} \mathrm{CO} 2 \rightarrow 1$ line) are represented by grey squares shaded according to the values printed on the pixel. The blue and red ${ }^{12} \mathrm{CO} 2 \rightarrow 1$ wing emission is given as contour lines: the solid lines with contours ranging from 15 to 55 by $10 \mathrm{~K} \mathrm{~km} \mathrm{~s}^{-1}$ and increasing line strength for increasing intensity, the dotted lines with contours ranging from 20 to 100 by $20 \mathrm{~K} \mathrm{~km} \mathrm{~s}^{-1}$.

at high southern Declination offsets (lower panels) with $\simeq+0.8 \mathrm{~km} \mathrm{~s}^{-1} / 0.1 \mathrm{pc}$, the gradient in the west is smaller (approximately $+0.3 \mathrm{~km} \mathrm{~s}^{-1} / 0.1 \mathrm{pc}$ ). North of S106 IR (DEC offsets $>20^{\prime \prime}$ ), the structure is less tilted and any gradients are rather small if evident at all. The velocity distribution is also not compatible with that of an expanding cavity (which was suggested by Little et al. 1995).

\section{Analysis and discussion}

\subsection{The dynamic scenario of the S106 region}

From the high angular resolution isotopomeric, multiline $\mathrm{CO}$ data presented in the previous section, we gained new insight on the small scale molecular cloud structure. This enables us to propose a scenario that explains the origin of the observed high-velocity emission distribution.

We attribute the dynamics of the molecular gas to the impact of the ionized wind of S106 IR, driving a shock into an inhomogeneous molecular cloud. In order to illustrate this and to discuss the most promiment features of S106, we selected images of four velocity planes of the ${ }^{13} \mathrm{CO} 2 \rightarrow 1$ emission and overlaid them in Fig. 10 on a $J H K$ picture of S106 taken with the Subaru ${ }^{2}$ telescope.

Panel a shows highly localized blue wing emission centered just east of S106 IR, panel d the more diffuse red counterpart 20" north-west and southeast of S106 IR. The fact that the blueshifted emission is much more pronounced indicates that the blueshifted part of the ionized wind drives into a denser region/dense clump on the near side of the molecular cloud, leading to larger masses of

\footnotetext{
${ }^{2}$ Subaru is an $8.2 \mathrm{~m}$ optical-infrared telescope at the summit of Mauna Kea, Hawaii, operated by the National Astronomical Observatory of Japan (NAOJ) with the support of the Ministry of Education, Culture, Sports, Science, and Technology.
}

swept-up material. This emission region is part of the East Clump, which wraps around S106 IR (with the denser part just east of S106 IR and less dense material in front and behind S106 IR). The high velocity emission hence marks where the ionized stellar wind hits the edge of the clump east of S106 IR in the fore- and background.

The dark lane, seen in panel b, appears to be a continuation of the East Clump to higher RA offsets, while the clump containing S106 FIR is linked with a bridge of emission to the East Clump, separating the optical lobes (also visible in Fig. 6 or Fig. 9). The CO emission distribution clearly shows that there is no smooth disk (see also Sect. 3), as it was already excluded by the observations of Barsony et al. (1989), and that the molecular gas is rather clumpy. The bipolar shape of the H II region then can be due to (i) an asymmetric stellar wind (Felli et al. 1984; Persson et al. 1988) or (ii) a very small circumstellar disk that extends within several AU of S106 IR (Bally et al. 1998).

The clumps could be the remnants of a toroid or disk, as it was suggested by Little et al. (1995), but there is no clear evidence for this assumption as reasoned from the position-velocity channel maps of Fig. 9. Moreover, the observed $\mathrm{CO}$ emission distribution can be explained exclusively by that of a clumpy molecular cloud. The impact of the $\mathrm{H}$ II region on the molecular cloud structure is clearly visible in panel c: east and west of S106 IR, the hot, ionized gas of the optical and radio lobes sweeps up the surrounding molecular gas and we see therefore a sharp gradient between the cavities and the molecular cloud.

The more diffuse and extended blue high-velocity wing (panel a) emission 150" west and east of S106 IR most likely results from a dispersed wind "leaking" through the gaps between individual molecular clumps. Panel d) shows red wing emission north and south of S106 IR, with the higher intensity contours closely following the 


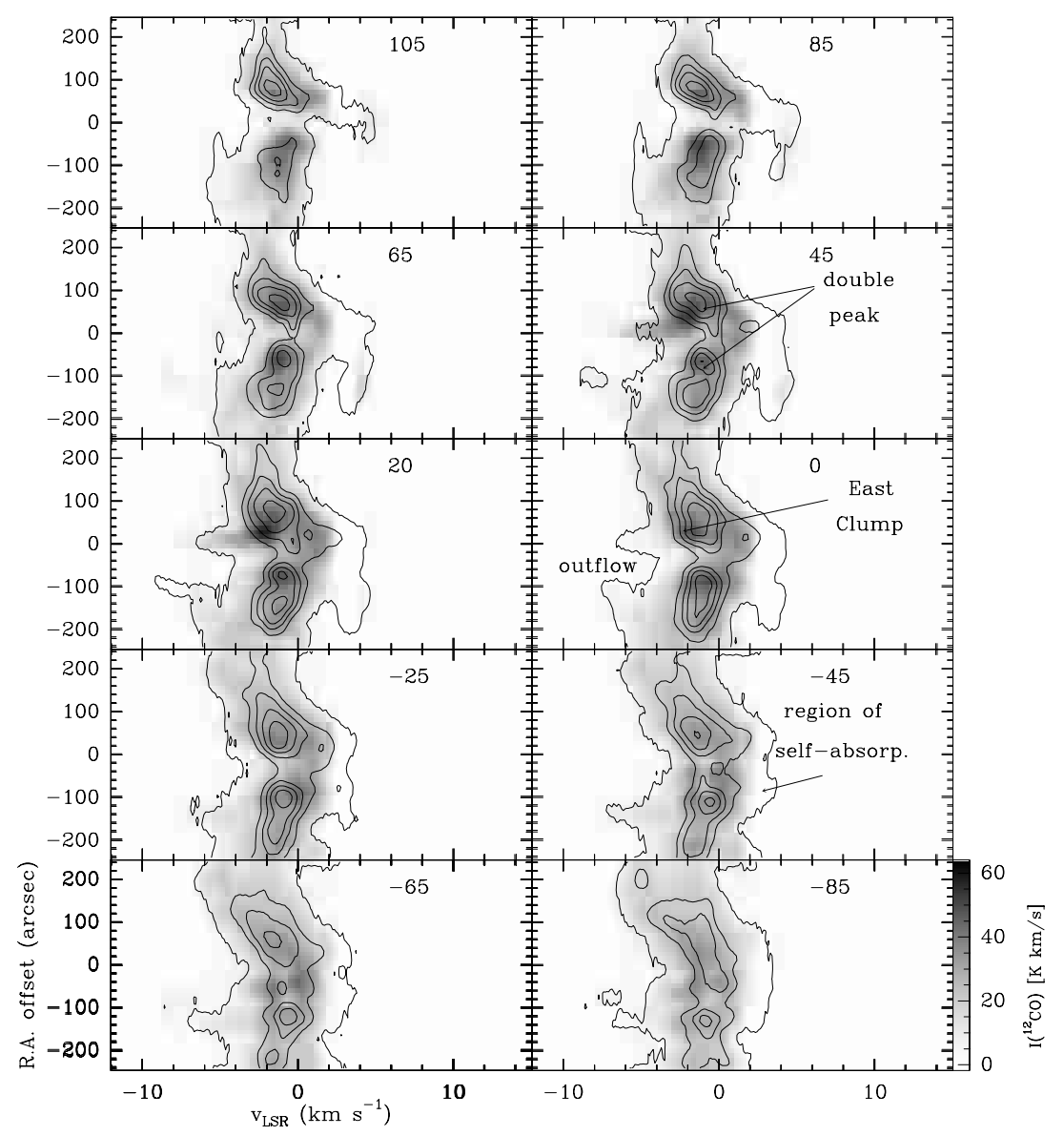

Fig. 9. Position-velocity channel maps of ${ }^{12} \mathrm{CO}$ and ${ }^{13} \mathrm{CO} 1 \rightarrow 0$ of the whole region observed with IRAM along cuts at constant Declination in steps of $20^{\prime \prime}$. (Due to the OFT mapping mode, the observing grids for the two maps are slightly different so that the Declination offset is an average value.) The ${ }^{12} \mathrm{CO} 1 \rightarrow 0$ emission is shown as grey scales with a wedge on the right hand side giving the intensity in $\mathrm{K} \mathrm{km} \mathrm{s}^{-1}$, the ${ }^{13} \mathrm{CO}$ emission as contours in the plot range 0.5 to $20.5 \mathrm{~K} \mathrm{~km} \mathrm{~s}^{-1}$ in steps of $5 \mathrm{~K} \mathrm{~km} \mathrm{~s}^{-1}$. Important features discussed in the text are labeled.

optical/IR lobes, which results from molecular material swept-up from the walls on the far side of the ionized cavity (Richer et al. 1993).

\subsection{The mass and clump-mass distribution of the molecular cloud}

\subsubsection{The total mass of the molecular cloud}

Since the KOSMA ${ }^{12} \mathrm{CO} 3 \rightarrow 2$ map provides the most complete coverage of the large scale molecular cloud, we use it in the following to estimate the total mass of the cloud complex. The integrated line intensity of the map leads to an estimate of $2000 M_{\odot}$ for the total mass and an average density of $1400 \mathrm{~cm}^{-3}$. We adopted a distance of $600 \mathrm{pc}$ and used the conversion factor (X-factor) of $2.3 \times 10^{20} \mathrm{~cm}^{-2} \mathrm{~K}^{-1}\left(\mathrm{~km} \mathrm{~s}^{-1}\right)^{-1}$ (Strong et al. 1988) for the conversion of the ${ }^{12} \mathrm{CO}$ line integrated intensity into $\mathrm{H}_{2}$ column densities. Even though this conversion factor was determined for the $1 \rightarrow 0$ transition of $\mathrm{CO}$, we adopt this value for the $3 \rightarrow 2$ line since the ratio of the line integrated intensities ${ }^{12} \mathrm{CO} 1 \rightarrow 0 / 3 \rightarrow 2$, using our KOSMA ${ }^{12} \mathrm{CO} 3 \rightarrow 2$ data and data from Bally \& Scoville (1982), is typically 1 (at a few positions it increases up to 1.4). Bally \& Scoville determined a mass of $1200 M_{\odot}$ from their ${ }^{13} \mathrm{CO}$ data, using a distance of $500 \mathrm{pc}$ and not including the lower intensity outer regions of the cloud.

We performed a clump identification on the spatially fully sampled KOSMA ${ }^{12} \mathrm{CO} 3 \rightarrow 2$ and IRAM ${ }^{13} \mathrm{CO} 2 \rightarrow 1$, ${ }^{13} \mathrm{CO} 1 \rightarrow 0$, and $\mathrm{C}^{18} \mathrm{O} 1 \rightarrow 0$ data sets. The latter cover $7 \%$ of the KOSMA ${ }^{12} \mathrm{CO} 3 \rightarrow 2$ map. We used the algorithm GAUSSCLUMPS, developed by Stutzki \& Güsten (1990) and discussed in Kramer et al. (1998a), to decompose three-dimensional data (two spatial axes and the velocity axis) into individual clumps, assuming Gaussian density and velocity distributions. Only clumps which are intrinsically (i.e., after deconvolution) larger than $50 \%$ of the spectral and spatial resolution were considered. The clump masses were calculated assuming optically thin emission and LTE (Local Thermodynamic Equilibrium) for the ${ }^{13} \mathrm{CO}$ and $\mathrm{C}^{18} \mathrm{O}$ lines (as described in, e.g., Schneider et al. 1998 or Herbertz et al. 1991) and a distance of 600 pc. The values for the excitation temperatures $T_{\text {ex }}$ and optical depths (for the optically thin lines) were taken from Sect. 4.3. (IRAM data) or determined from the KOSMA $3 \rightarrow 2$ map. 

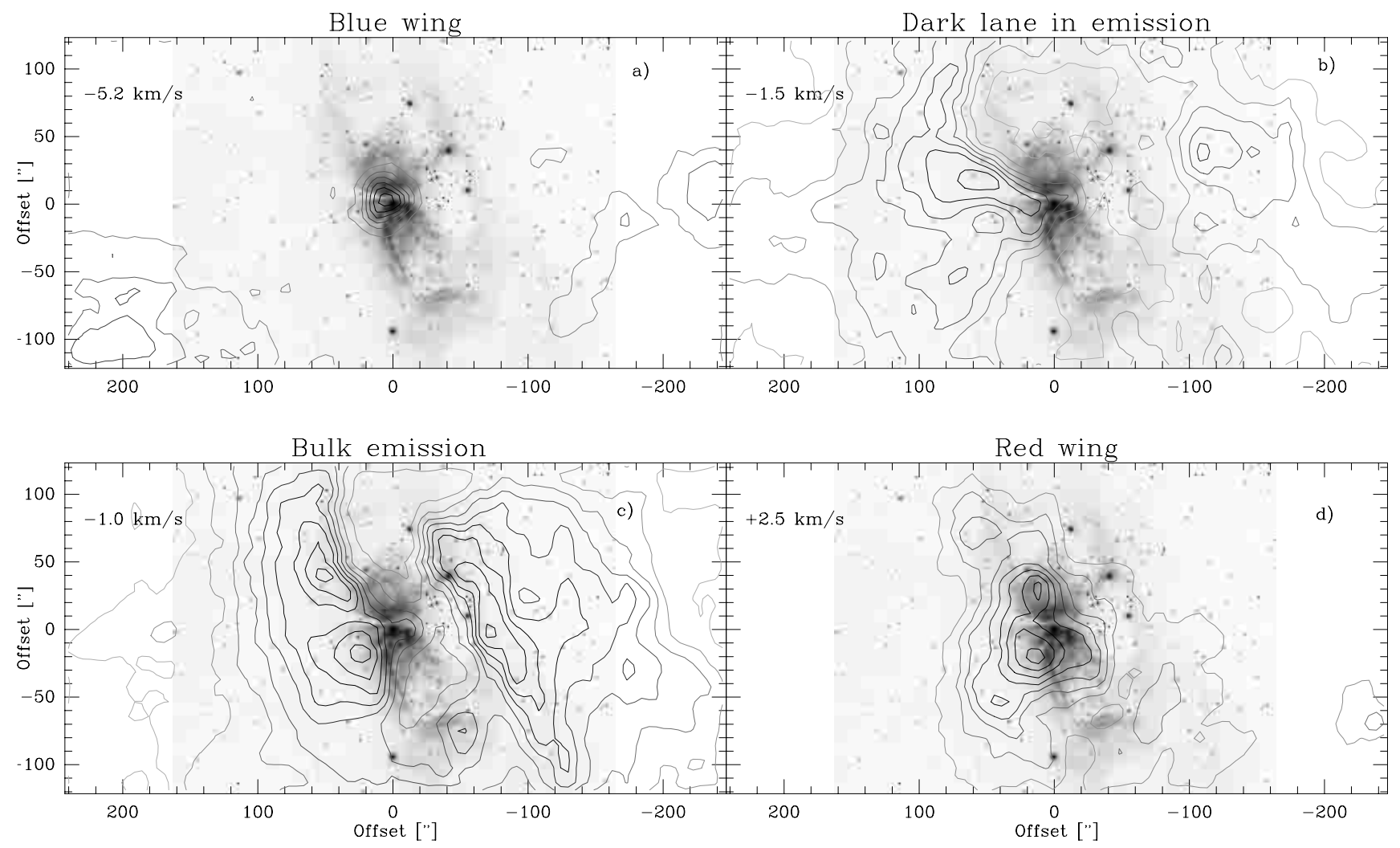

Fig. 10. Four different velocity planes of the ${ }^{13} \mathrm{CO} 2 \rightarrow 1$ emission at $11^{\prime \prime}$ angular resolution (representing the most prominent features of the region) are overlaid on the $J H K$ image from the SUBARU telescope. The contour levels are as follows (notation start/end/step): a) $0.7 / 11.2 / 0.7 \mathrm{~K} \mathrm{~km} \mathrm{~s}^{-1}$, b) $1.4 / 22.4 / 1.4 \mathrm{Kkm} \mathrm{s}^{-1}$, c) $1.75 / 28 / 1.75 \mathrm{Kkm} \mathrm{s}^{-1}$, d) $1.05 / 16.8 / 1.05 \mathrm{Kkm} \mathrm{s}{ }^{-1}$.

Figure 11 shows a log-log plot of the combined clumpmass spectrum obtained from the 4 molecular tracers. The clumps from the IRAM data are scaled to match the KOSMA histogram according to the procedure described in Schneider et al. (1998) and Heithausen et al. (1998). For all data sets, the number of clumps increases with decreasing mass until a turn over point, the completeness limit, is reached which results from finite angular resolution and limited Signal-to-Noise $(S / N)$ ratio of the data.

\subsubsection{Clump-mass distribution}

The ${ }^{12} \mathrm{CO} 3 \rightarrow 2$ clump-mass spectrum covers a mass range of 1 to $350 M_{\odot}$ on a size-scale of clumps between 0.12 to $0.9 \mathrm{pc}$. In contrast, the higher resolution IRAM maps are more sensitive to the small scale structure and cover a mass interval from approximately 0.1 to $60 M_{\odot}$ with clump sizes from 0.06 to $0.2 \mathrm{pc}$. Thus, due to the lower angular resolution of the KOSMA map, the ${ }^{12} \mathrm{CO} 3 \rightarrow 2$ emission is decomposed into larger, more massive clumps and a lower number of small clumps. The turnover in the clumpmass spectra and the lowest mass identified are different in the individual IRAM data sets because (i) the ${ }^{13} \mathrm{CO} 2 \rightarrow 1$ map has a factor 2 higher angular resolution compared to the isotopomeric $\mathrm{CO} 1 \rightarrow 0$ maps, and therefore traces the smallest structures and less massive clumps, and (ii) the maps have different Signal-to-Noise ratios (the best quality map is that of ${ }^{13} \mathrm{CO} 1 \rightarrow 0$ with a peak $S / N$ ratio of
110 , followed by ${ }^{13} \mathrm{CO} 2 \rightarrow 1(S / N=67)$ and $\mathrm{C}^{18} \mathrm{O} 1 \rightarrow 0$ $(S / N=25)$. Accordingly, a higher temperature threshold was used for the $\mathrm{C}^{18} \mathrm{O}$ clump identification.

A fit to the power law function $\mathrm{d} N / \mathrm{d} M \propto M^{-\alpha}$, in which $\mathrm{d} N$ denotes the number of clumps within the mass interval $\mathrm{d} M$, for masses above the completeness limit yields the same value of the clump-mass spectral index for each data set within the error (typically 1 to $2 \%$ ): $\alpha=1.72$ for ${ }^{12} \mathrm{CO} 3 \rightarrow 2, \alpha=1.72$ for ${ }^{13} \mathrm{CO} 2 \rightarrow 1, \alpha=1.74$ for ${ }^{13} \mathrm{CO} 1 \rightarrow 0$, and $\alpha=1.69$ for $\mathrm{C}^{18} \mathrm{O} 1 \rightarrow 0$. The run of the power law function with the rounded value of $\alpha=1.7$ is indicated as a straight line in Fig. 10. The common clump-mass spectral index shows that the structures are self-similar at least over a length-scale from 0.06 to $0.9 \mathrm{pc}$, the sizes of the smallest and largest clump. This result is remarkable, considering that we used molecular lines with different opacities, tracers of different excitation conditions and because the spatial and kinematic structure of S106 is governed by the interaction with the stellar wind.

The observed clump-mass distribution is typical for Giant Molecular Clouds and the clump-mass spectral index of 1.7 agrees with results from other molecular clouds, typically 1.4 to 1.9 (Kramer et al. 1998a; Simon et al. 2001), in a broad range of different interstellar clouds and using different molecular tracers. 


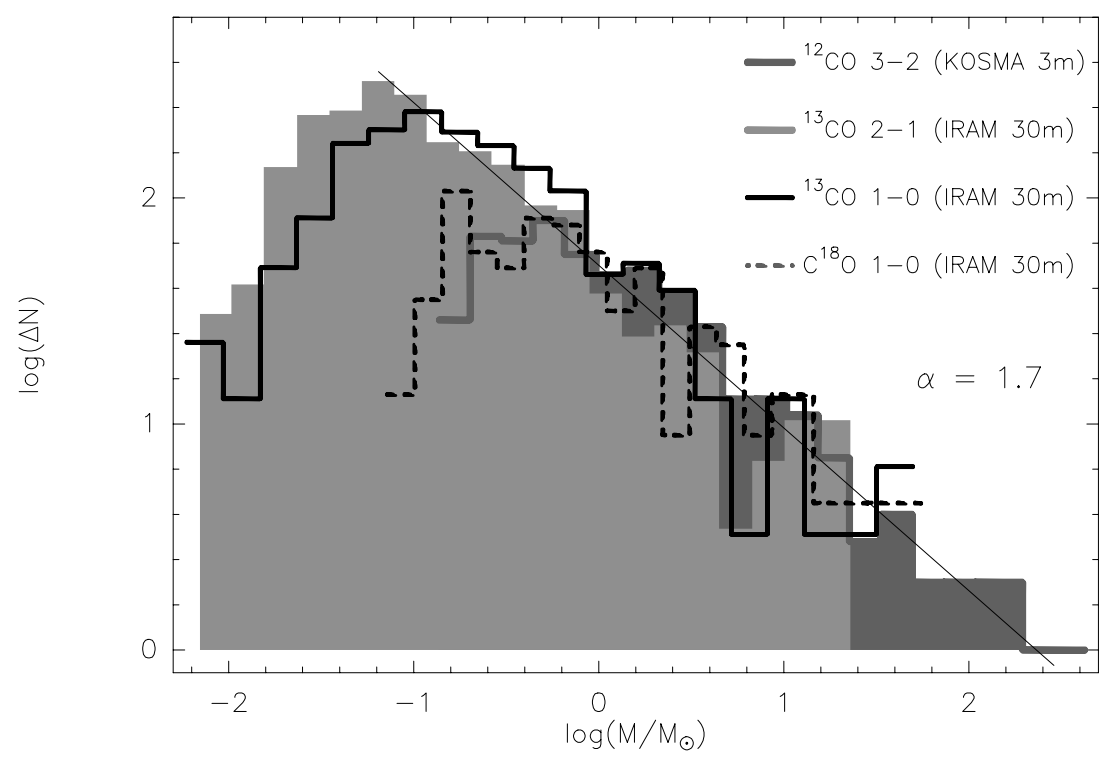

Fig. 11. Combined clump-mass spectrum derived from the KOSMA ${ }^{12} \mathrm{CO} 3 \rightarrow 2$ and IRAM ${ }^{13} \mathrm{CO} 2 \rightarrow 1,{ }^{13} \mathrm{CO} 1 \rightarrow 0$ and $\mathrm{C}^{18} \mathrm{O} 1 \rightarrow 0$ data sets showing the number of clumps $\mathrm{d} N$ in the mass interval $\mathrm{d} M$. The straight line indicates the fit to the power law function $\mathrm{d} N / \mathrm{d} M \propto M^{-\alpha}$ with $\alpha=1.7$.

Table 2. The excitation conditions for the bulk emission of the cloud $\left(-1 \mathrm{~km} \mathrm{~s}^{-1}\right.$ component) along the cut through S106 IR as determined from CO $1 \rightarrow 0$ observations. The position $\left(20^{\prime \prime}, 0\right)$ corresponds approximately to the location of the East Clump and $\left(-20^{\prime \prime}, 0\right)$ to that of S106 FIR. $T_{\text {ex }}$ is the excitation temperature determined from ${ }^{12} \mathrm{CO} 1 \rightarrow 0$ data, $\tau\left({ }^{13} \mathrm{CO}\right)$ the optical depth of the ${ }^{13} \mathrm{CO} 1 \rightarrow 0$ line, ${ }^{13} \mathrm{CO} / \mathrm{C}^{18} \mathrm{O}$ the $1 \rightarrow 0$ line ratio, $N\left({ }^{13} \mathrm{CO}\right)$ the ${ }^{13} \mathrm{CO}$ column density, $N\left({ }^{13} \mathrm{CO}\right) / N\left(\mathrm{C}^{18} \mathrm{O}\right)$ the ratio of the column densities, and $N\left(\mathrm{H}_{2}\right)$ the $\mathrm{H}_{2}$ column density determined from ${ }^{13} \mathrm{CO} 1 \rightarrow 0$.

\begin{tabular}{|c|c|c|c|c|c|c|c|c|}
\hline \multicolumn{3}{|c|}{ Offset } & $\begin{array}{l}T_{\mathrm{ex}} \\
(\mathrm{K})\end{array}$ & $\tau\left({ }^{13} \mathrm{CO}\right)$ & $\begin{array}{c}{ }^{13} \mathrm{CO} / \mathrm{C}^{18} \mathrm{O} \\
\text { line ratio }\end{array}$ & $\begin{array}{c}N\left({ }^{13} \mathrm{CO}\right) \\
\left(10^{17} \mathrm{~cm}^{-2}\right)\end{array}$ & $N\left({ }^{13} \mathrm{CO}\right) / N\left(\mathrm{C}^{18} \mathrm{O}\right)$ & $\begin{array}{c}N\left(\mathrm{H}_{2}\right) \\
\left(10^{22} \mathrm{~cm}^{-2}\right)\end{array}$ \\
\hline 80 & 0 & & 40 & 0.6 & 4 & 0.8 & 4 & 3.8 \\
\hline 40 & 0 & East & 54 & 0.3 & 4 & 0.6 & 3 & 2.8 \\
\hline 20 & 0 & & 43 & 0.6 & 6 & 0.7 & 4 & 3.3 \\
\hline 0 & 0 & IR & $>32$ & $<0.8$ & 6 & $<0.6$ & 6 & $<2.8$ \\
\hline-20 & 0 & FIR & $>29$ & $<0.5$ & 8 & $<0.4$ & 8 & $<1.9$ \\
\hline-40 & 0 & & 27 & 0.4 & 11 & 0.5 & 17 & 2.4 \\
\hline-80 & 0 & & 49 & 0.6 & 7 & 1.5 & 12 & 7.1 \\
\hline
\end{tabular}

\subsection{The excitation conditions along a cut through S106 IR}

In order to estimate the excitation conditions throughout the S106 region including the interface of the molecular cloud to the $\mathrm{H}$ II region, we perform a straight-forward LTE analysis, using ${ }^{12} \mathrm{CO},{ }^{13} \mathrm{CO}$, and $\mathrm{C}^{18} \mathrm{O} \quad 1 \rightarrow 0$ data. We use these lower frequency observations since they are less affected by the error beam (see Sect. 2). The excitation temperature $\left(T_{\mathrm{ex}}\right)$ is calculated by assuming an optically thick ${ }^{12} \mathrm{CO}$ line and optically thin ${ }^{13} \mathrm{CO}$ and $\mathrm{C}^{18} \mathrm{O} 1 \rightarrow 0$ line (Little et al. 1995). Since we assume LTE the excitation temperatures for all isotopomers are equal. With the resulting opacities and the line integrated ${ }^{13} \mathrm{CO}$ and $\mathrm{C}^{18} \mathrm{O}$ intensities in $\mathrm{K} \mathrm{km} \mathrm{s}^{-1}$, we derive the ${ }^{13} \mathrm{CO}$ and $\mathrm{C}^{18} \mathrm{O}$ column density and $\mathrm{H}_{2}$ column density as described in, e.g., Frerking et al. (1982) and Dickman (1978).

Table 2 gives an overview of the physical conditions of the bulk emission of the molecular cloud (at a velocity of
$-1.0 \mathrm{~km} \mathrm{~s}^{-1}$ ) along an east-west cut from $+80^{\prime \prime}$ to $-80^{\prime \prime}$. The results especially at the positions of S106 IR and S106 FIR should be treated with caution since the ${ }^{12} \mathrm{CO}$ line shows self-absorption effects around $-1 \mathrm{~km} \mathrm{~s}^{-1}$. The excitation temperatures are therefore lower limits and the opacities and column densities upper limits. Keeping that in mind, we find that the maximum values for $T_{\mathrm{ex}}$ (around $45 \mathrm{~K}$ ) are in the eastern part of the molecular cloud. The opacity of the ${ }^{13} \mathrm{CO}$ line is generally smaller than 1 with a minimum of 0.25 at $\left(40^{\prime \prime}, 0\right)$ and a maximum of 0.8 at the position of S106 IR. A direct comparison between S106 FIR, S106 IR, and the East Clump shows that the ${ }^{13} \mathrm{CO}$ line remains optically thin everywhere at an excitation temperature of around $30 \mathrm{~K}$ for S106 IR and the East Clump and a slightly higher value $(40 \mathrm{~K})$ for S106 FIR. Generally, the derived values for excitation temperatures and column densities agree well with the ones given by Little et al. (1995), obtained from JCMT observations (not 
affected by any error beam pick-up). From the average $\mathrm{H}_{2}$ column density of $3.4 \times 10^{22} \mathrm{~cm}^{-2}$, we derive an average density of $9.1 \times 10^{3} \mathrm{~cm}^{-3}$ of the cloud core region (from its projected $\sim 400^{\prime \prime}$ size in the CO maps and assuming the same extent along the line-of-sight).

We determine the ${ }^{13} \mathrm{CO} / \mathrm{C}^{18} \mathrm{O}$ line and column density ratio in order to look for systematic changes of this ratio along the cut. The ${ }^{13} \mathrm{CO} / \mathrm{C}^{18} \mathrm{O}$ ratio is expected to increase in regions of high incident UV radiation due to effective self-shielding of the more abundant species ${ }^{13} \mathrm{CO}$. If the cloud structure is clumpy on small scales, this effect is even enhanced since small clumps have even less shielding column for the $\mathrm{C}^{18} \mathrm{O}$ molecule to survive. The ${ }^{13} \mathrm{CO} / \mathrm{C}^{18} \mathrm{O}$ ratio is thus also correlated with the sizes of the clumps. (See Zielinsky et al. 2000 for a detailed discussion of these issues.) In our case, the ${ }^{13} \mathrm{CO} / \mathrm{C}^{18} \mathrm{O}$ brightness ratio along the cut takes values between 4 and 11 which is close to the natural isotopic abundance of $\sim 7$. Since the ${ }^{13} \mathrm{CO}$ column densities were determined including the line opacities, the column density ratio is a more reliable tracer of changes in the ${ }^{13} \mathrm{CO} / \mathrm{C}^{18} \mathrm{O}$ abundance ratio. We find values in the range from 3 to 8 for the positions $-20^{\prime \prime}$ to $80^{\prime \prime}$, and significantly larger at $-40^{\prime \prime}$ and $-80^{\prime \prime}$ with ratios of 17 and 12 , respectively. The low ratios observed towards the East Clump suggest that the bulk of $\mathrm{CO}$ emission originates in rather large clumps and that selective self-shielding is only important in a thin surface layer of the clumps and close to the interface to the HiI region.

To further analyze the correlation between changes in the ${ }^{13} \mathrm{CO} / \mathrm{C}^{18} \mathrm{O}$ ratio and the $\mathrm{UV}$ radiation, we show in Fig. 12 an image of the ${ }^{13} \mathrm{CO} / \mathrm{C}^{18} \mathrm{O} 1 \rightarrow 0$ integrated intensity ratio for a $0.5 \mathrm{~km} \mathrm{~s}^{-1}$ wide channel centered on $v=-1 \mathrm{~km} \mathrm{~s}^{-1}$. The high ratios at the outer cloud boundaries are due to noise in the $\mathrm{C}^{18} \mathrm{O}$ emission, but the low ratios toward the eastern and western $\mathrm{CO}$ peaks indicate moderate optical depths for the ${ }^{13} \mathrm{CO}$ line and no depletion (i.e., effective shielding) of the $\mathrm{C}^{18} \mathrm{O}$ molecule.

The ratios are highest where $\mathrm{CO}$ is not depleted completely and the $[\mathrm{C} \mathrm{II}]$ emission is intense: (1) towards the bridge of emission across S106 IR, (2) along the southwestern cavity wall of the $\mathrm{H}$ II region, following the tongue of [C II] emission seen in Fig. 3, and (3) towards the tip of the southern optical lobe. In these regions, we see a steep gradient of the ratio with distance away from the UV source and the $\mathrm{H}$ in region, supporting our assessment that the bulk of the emission originates from rather large, well shielded clumps and that selective self-shielding of ${ }^{13} \mathrm{CO}$ is only important close to the $\mathrm{H}$ in region.

\subsection{Multi line analysis with an escape probability model}

A more sophisticated approach to determine the physical parameters of the molecular cloud or clump is to solve the radiative transfer equation explicitly but using the approximation that the level populations are independent of the position in the cloud. Then, the probability of a photon to leave the cloud without being absorbed depends on the geometry considered and on the value of the optical depth. Here, we use an escape probability formalism for a homogeneous, spherical cloud (Stutzki \& Winnewisser 1985) which computes line intensities, integrated intensities, rotational temperatures, and optical depths in a user-defined data cube spanned by the molecular column density $N(\mathrm{CO}), \mathrm{H}_{2}$ density $n\left(\mathrm{H}_{2}\right)$, and kinetic temperature $T_{\text {kin }}$. The collision rates were taken from Flower \& Launay (1985).

We use the observed ${ }^{13} \mathrm{CO} 6 \rightarrow 5 /{ }^{13} \mathrm{CO} 3 \rightarrow 2$ and ${ }^{13} \mathrm{CO}$ $2 \rightarrow 1 /{ }^{13} \mathrm{CO} 1 \rightarrow 0$ line ratios (all data on or smoothed to an angular resolution of $80^{\prime \prime}$ ) to constrain a parameter space for $N\left({ }^{13} \mathrm{CO}\right), n\left(\mathrm{H}_{2}\right)$, and $T_{\text {kin }}$ since the beam filling factor cancels out to first order by using line ratios. We focus on only one position (S106 IR) since the 80" KOSMA beam comprises the East Clump and S106 FIR. In addition, the variation of $T_{\mathrm{ex}}$ and $\tau$ in our LTE analysis across this area is not large, as it was shown in Sect. 4.3, so that the results for S106 IR should yield a good constraint on the excitation conditions in the molecular cloud core.

The ${ }^{13} \mathrm{CO} 6 \rightarrow 5 /{ }^{13} \mathrm{CO} 3 \rightarrow 2$ peak line ratio of 0.7 together with a ${ }^{13} \mathrm{CO} 2 \rightarrow 1 /{ }^{13} \mathrm{CO} 1 \rightarrow 0$ ratio of 1.2 is only consistent with escape probability models of kinetic temperatures between 40 and $100 \mathrm{~K}$. We adopt an average excitation temperature of $40 \mathrm{~K}$ from the LTE analysis to derive a hydrogen density of $9 \times 10^{4} \mathrm{~cm}^{-3}$ and a ${ }^{13} \mathrm{CO}$ column density of $1.3 \times 10^{17} \mathrm{~cm}^{-2}$ from the corresponding escape probability model at $T_{\text {kin }}=40 \mathrm{~K}$. The corresponding $\mathrm{H}_{2}$ column density then is $6.1 \times 10^{22} \mathrm{~cm}^{-2}$. This value is only a factor 2 larger than the $\mathrm{H}_{2}$ column density determined by the LTE analysis, indicating that the beam is filled rather homogeneously. We can now determine a typical clump size by $d=N\left(\mathrm{H}_{2}\right) / n_{\text {local }}$ with the local density of $9 \times 10^{4} \mathrm{~cm}^{-3}$ and the column density $N\left(\mathrm{H}_{2}\right)$ given by the model. The typical diameter of a clump then is $0.22 \mathrm{pc}$ $(1.2)$, which is rather large and implies a predominantly homogeneous structure of the material in that region. This finding supports the scenario of large clumps with high area filling found from the comparison of the ${ }^{13} \mathrm{CO}$ to $\mathrm{C}^{18} \mathrm{O}$ line and column density ratios. The co-existence of very small, high density clumps, however, is not excluded. In fact, the clump decomposition of the IRAM CO data indicates substructure (Sect. 4.2 ) below 0.22 pc. We therefore derive beam and volume filling factors in the following section to address this issue.

\subsection{Beam and volume filling factors}

The beam filling factor $F_{\mathrm{b}}$ is calculated from $F_{\mathrm{b}}=$ $T_{\text {mb }} / T_{\text {model }}$ with the observed main beam brightness temperature of the respective transition $\left(T_{\mathrm{mb}}\right)$ and the temperature $T_{\text {model }}$ which is obtained from the escape probability model, given the kinetic temperature, column and volume densities (Beuther et al. 2000). $F_{\mathrm{b}}$ varies between $40 \%$ for the ${ }^{13} \mathrm{CO} 6 \rightarrow 5$ and ${ }^{13} \mathrm{CO} 3 \rightarrow 2$ transitions, $50 \%$ 


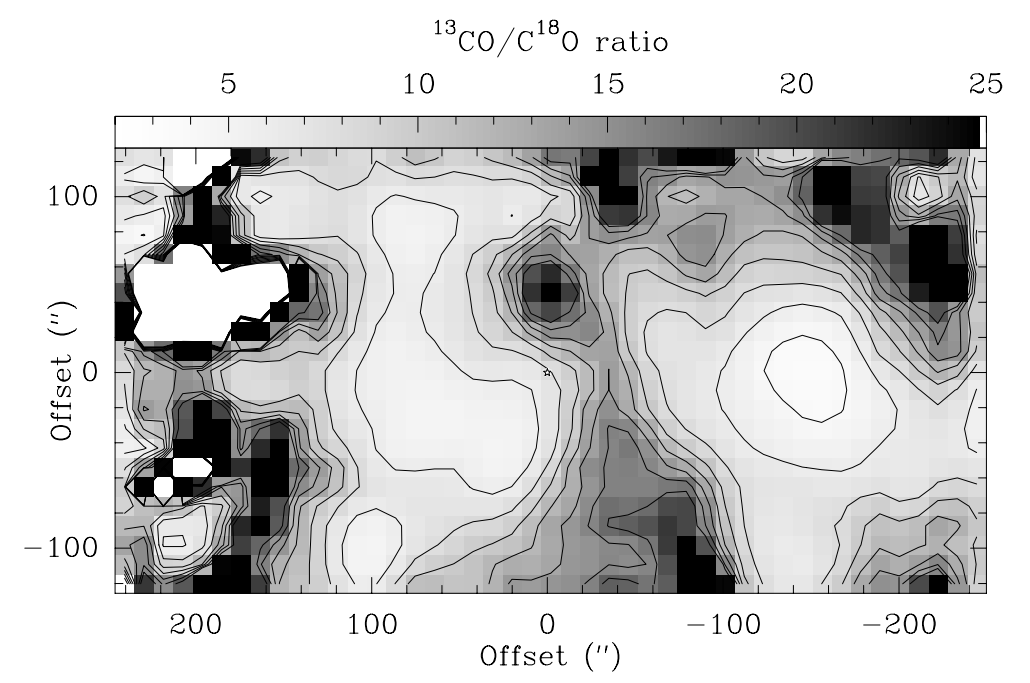

Fig. 12. Map of the ${ }^{13} \mathrm{CO} / \mathrm{C}^{18} \mathrm{O} 1 \rightarrow 0$ integrated intensity ratio for a $0.5 \mathrm{~km} \mathrm{~s}^{-1}$ wide channel centered on $v=-1 \mathrm{~km} \mathrm{~s}{ }^{-1}$. The plot range is given on top of the image. Contour lines are drawn for ratios between 4 and 18 in steps of 2 .

for ${ }^{13} \mathrm{CO} 2 \rightarrow 1$, and $60 \%$ for ${ }^{13} \mathrm{CO} 1 \rightarrow 0$. The volume filling factor of clumps is a measure for the small scale structure in a cloud and is defined as $F_{\mathrm{v}}=\left(n_{\text {aver }}-n_{\text {ic }}\right) /\left(n_{\text {local }}-n_{\text {ic }}\right)$. In our case the average density is $n_{\text {aver }}=9.1 \times 10^{3} \mathrm{~cm}^{-3}$, determined from the LTE analysis, and the local density is $n_{\text {local }}=9 \times 10^{4} \mathrm{~cm}^{-3}$ obtained from the Escape Probability Model. The density of the interclump medium, $n_{\text {ic }}$ cannot be deduced in a straight-forward manner. Due to the rather high local and average densities, however, the volume filling factor remains 0.1 (10\%) for a density regime of 50 to $600 \mathrm{~cm}^{-3}$ as a realistic range for $n_{\text {ic }}$. Our value of $10 \%$ (typical values are a few to $20 \%$ ) implies a rather low volume filling and therefore small scale structure of the molecular material. This result is not in contradiction to the results obtained in Sects. 4.3 and 4.4 that the low- $J$ CO emission arises from a predominantly homogeneous medium (clumps on a size-scale larger than $0.2 \mathrm{pc}$ $\left(1^{\prime}\right)$, with a density around $10^{4} \mathrm{~cm}^{-3}$ and a temperature of $\left.T_{\text {kin }}=40 \mathrm{~K}\right)$ if we postulate the existence of embedded, small $(\ll 0.2 \mathrm{pc})$, high density $\left(n \simeq 10^{5} \mathrm{~cm}^{-3}\right)$ and presumably warm clumps with a low volume filling factor. In this picture, the observed ${ }^{13} \mathrm{CO} \quad 6 \rightarrow 5$ emission arises from these high density clumps, since the critical density of the $6 \rightarrow 5$ transition is much larger than the density for the large clumps, derived from the CO line analysis. In Part II of the series, we will support this scenario of a two-component gas phase by modelling the observed $\mathrm{CO}$ line intensities as well as [C II] and [O I] fine structure lines to derive the temperature of the warm PDR gas and the intensity of the incident UV flux.

\section{Summary}

We presented a spectroscopic study of the molecular cloud associated with S106 in $\mathrm{mm}$ and submm rotational transitions of CO isotopomers obtained with the KOSMA $3 \mathrm{~m}$ and IRAM $30 \mathrm{~m}$ radiotelescopes. The main results of this study are summarized below as follows.
1. The KOSMA ${ }^{12} \mathrm{CO} 3 \rightarrow 2$ map reveals the large scale distribution of molecular gas $\left(24^{\prime} \times 25^{\prime}\right.$ or $4.2 \times 4.4 \mathrm{pc}$ at a distance of $600 \mathrm{pc})$. The total mass is $2000 M_{\odot}$ and the average density $1.4 \times 10^{3} \mathrm{~cm}^{-3}$.

2. The IRAM ${ }^{12} \mathrm{CO}$ and ${ }^{13} \mathrm{CO} 2 \rightarrow 1$, and ${ }^{12} \mathrm{CO},{ }^{13} \mathrm{CO}$ and $\mathrm{C}^{18} \mathrm{O} 1 \rightarrow 0$ observations focus on the molecular cloud core around S106 IR $\left(8^{\prime} \times 4^{\prime}\right)$. We identify two well defined emission maxima east and west of S106 IR separated by $3^{\prime}$, with the $\mathrm{H}$ II region outlined by a lack of emission. On a smaller scale, we distinguish two dense molecular clumps east (East Clump) and west (S106 FIR, not resolved) of S106 IR.

An analysis of the line profiles shows that there is self-absorption in ${ }^{12} \mathrm{CO}$ around $\mathrm{S} 106 \mathrm{IR}$ at $-1 \mathrm{~km} \mathrm{~s}^{-1}$ (the velocity of the bulk emission of the cloud) as well as at $+3 \mathrm{kms}^{-1}$, partly hiding the redshifted (from 2 to $6 \mathrm{~km} \mathrm{~s}^{-1}$ ) counterpart of the blue wing emission.

3. The high angular resolution position-velocity and velocity channel maps of CO clearly show that there is no smooth molecular disk on a size-scale of $6^{\prime}$. Our observations also do not support an expanding cavity. A fragmented toroid, however, cannot be completely excluded, but the CO emission distribution can be exclusively explained by a fragmented molecular cloud.

The emission distribution of the high-velocity gas can be explained by the ionized wind of S106 IR, driving a shock into the clumpy molecular cloud.

4. The ${ }^{12} \mathrm{CO} \quad 3 \rightarrow 2$ map and the ${ }^{13} \mathrm{CO}$ and $\mathrm{C}^{18} \mathrm{O} \quad 2 \rightarrow 1$ and $1 \rightarrow 0$ maps were decomposed into clumps. We find a common clump-mass spectral index of $\alpha=1.7$, which indicates self-similarity of the detected structures over lengthscales from 0.06 to 0.9 parsec.

5. We discuss the excitation conditions along a cut through the molecular cloud and S106 IR within the framework of an LTE and Escape Probability analysis. We find that the kinetic gas temperature is typically $40 \mathrm{~K}$ and that the ${ }^{13} \mathrm{CO}$ lines are optically thin. The average density of the cloud in the core region is $9.1 \times 10^{3} \mathrm{~cm}^{-3}$ and the local 
density $9 \times 10^{4} \mathrm{~cm}^{-3}$, leading to a volume filling factor of $10 \%$. Since the typical size-scale of the clumps is $0.22 \mathrm{pc}$ $(1.2)$ and the observed ${ }^{13} \mathrm{CO} / \mathrm{C}^{18} \mathrm{O}$ line and column density ratios reflect the natural isotopic abundance, we derive the following scenario for the molcular cloud: very small ( $\ll 0.2 \mathrm{pc}$ ), high-density clumps with a small volume filling factor coexist with rather homogeneous, low-density and spatially extended clumps.

Acknowledgements. The KOSMA 3 m submillimeter telescope at the Gornergrat-Süd is operated by the University of Cologne in collaboration with Bonn University, and supported by special funding from the Land NRW. The observatory is administered by the International Foundation Gornergrat and Jungfraujoch.

We thank the referee, Dr. R. Gehrz, for useful suggestions to clarify and improve the manuscript.

\section{References}

Bally, J., \& Scoville, N. Z. 1982, ApJ, 255, 497

Bally, J., Yu, K. C., Rayner, J., \& Zinnecker, H. 1998, AJ, 116(4), 1868

Barsony, M., Scoville, N. Z., Bally, J., \& Claussen, M. J. 1989, ApJ, 343, 212

Beuther, H., Kramer, C., Deiss, B., \& Stutzki, J. 2000, A\&A, 362,1109

Bieging, J. H. 1984, ApJ, 286, 591

Bontemps, S. 2001, in The Origins of Stars and Planets: The VLT View, ESO Astrophysics Symp., ed. J. Alves, \& M. McCaughrean, Garching 24-27 April 2001

Bontemps, S., Andre, P., Kaas, A., et al. 2001, A\&A, 372, 173

Churchwell, E., \& Bieging, J. H. 1982, ApJ, 258, 515

Dickman, R. L. 1978, ApJS, 37, 407

Eiroa, C., Elsässer, H., \& Lahulla, J. F. 1979, A\&A, 74, 89

Felli, M., Staude, H. J., Reddmann, T., et al. 1984, A\&A, 135, 261

Flower, D., \& Launay, J. 1985, MNRAS, 214, 271

Frerking, M. A., Langer, W. D., \& Wilson, R. W. 1982, ApJ, 262,590

Furuya, A. R., Kitamura, Y., Claussen, M., et al. 1999, AAS, 194,5013

Garcia-Burillo, S., Guelin, M., \& Cernicharo, J. 1993, A\&A, 274,123

Gehrz, R., Grasdalen, G., Castelaz, M., et al. 1982, ApJ, 254, 550

Graf, U. U., Haas, S., Honingh, C., et al. 1998, Proc. SPIE, 3357,159

Greve, A., Kramer, C., \& Wild, W. 1998, A\&A, 133, 271

Grossmann, E. 1989, Atmosph. Transmission Software, User's Manual (Univ. of Texas, Austin)

Heithausen, A., Bensch, F., Stutzki, J., Falgarone, E., \& Panis, J. F. 1998, A\&A, 331, 65
Herbertz, R., Ungerechts, H., \& Winnewisser, G. 1991, A\&A, 249,483

Hippelein, H., \& Muench, G. 1981, A\&A, 99, 248

Hodapp, K., \& Rayner, J. 1991, AJ, 102, 1108

Knödlseder, J. 2000, A\&A, 360, 539

Kramer, C., Stutzki, J., Röhrig, R., \& Corneliussen, U. 1998a, A\&A, 329, 249

Kramer, C., Degiacomi, C., Graf, U. U., et al. 1998b, SPIE 1998, Kona/Hawaii

Kramer, C., Beuther, H., Simon, R., Stutzki, J., \& Winnewisser, G. 1999, Imaging at radio through submillimetre wavelength, ed. J. G. Mangum, \& S. J. E. Radford, Astronomical Society of the Pacific, CF-217

Lada, C. J. 1987, in Star Forming Regions, IAU Symp., 115, 1

Little, L., MacDonald, G. H., Riley, P. W., \& Metheson, D. N. 1979, MNRAS, 188, 429

Little, L., Kelly, M., Habing, R., \& Millar, T. 1995, MNRAS, 277,307

Loushin, R., Crutcher, R. M., \& Bieging, J. H. 1990, ApJ, 362, L67

Lucas, R., Le Squéren, A., Kazès, I., \& Encrenaz, P. J. 1978, A\&A, 66, 155

Mezger, P. G., Chini, R., Kreysa, E., \& Wink, J. E. 1987, A\&A, 182,127

Persson, S. E., McGregor, P. J., \& Campbell, B. 1988, ApJ, 326,339

Price, S. D., Egan, M. P., Shipman, R. F., Cohen, M., \& Moshir, M. 1998, in The Impact of Near-Infrared Sky Surveys on Galactic and Extragalactic Astronomy, ed. N. Epchtein (Astrophysics and space science library), 230, 17

Reifenstein, E. C., Wilson, T. L., Burke, B. F., Mezger, P. G., \& Altenhoff, W. J. 1970, A\&A, 4, 357

Richer, J., Padman, R., Ward-Thompson, D., Hills, R. E., \& Harris, A. I. 1993, MNRAS, 262, 839

Schieder, R., Tolls, V., \& Winnewisser, G. 1989, Exp. Astron., 1,101

Schneider, N., Stutzki, J., Winnewisser, G., et al. 1998, A\&A, 335, 1049

Schneider, N. 1999a, ESA SP-427, 755

Schneider, N. 1999b, Proc. of the 3rd Cologne-Zermatt Symposium, ed. V. Ossenkopf, J. Stutzki, \& G. Winnewisser (GCA-Verlag Herdecke)

Simon, R., Jackson, J. M., Clemens, D. M., Bania, T. M., \& Heyer, M. H. 2001, ApJ, 551, 747

Simon, M., \& Fischer, J. 1982, BAAS, 14, 925

Solf, J., \& Carsenty, U. 1982, A\&A, 113, 142

Staude, H. J., Lenzen, R., Dyck, H. M., \& Schmidt, G. D. 1983, ApJ, 255, 95

Strong, J., Bloemen, J., Dame, T., et al. 1988, A\&A, 207, 1

Stutzki, J., Ungerechts, H., \& Winnewisser, G. 1982, A\&A, 111,201

Stutzki, J., \& Winnewisser, G. 1985, A\&A, 144, 13

Stutzki, J., \& Güsten, R. 1990, ApJ, 356, L63

van den Ancker, M., Tielens, A. G. G. M., \& Wesselius, P. R. 1999, A\&A, 358, 1035

Zielinsky, M., Stutzki, J., \& Störzer, H. 2000, A\&A, 358, 723 